Research Article

\title{
Modeling and Risk Analysis Using Parametric Distributions with an Application in Equity-Linked Securities
}

\author{
Sun-Yong Choi ${ }^{1}{ }^{1}$ and Ji-Hun Yoon $\mathbb{D i D}^{2}$ \\ ${ }^{1}$ Department of Financial Mathematics, Gachon University, Gyeoggi 13120, Republic of Korea \\ ${ }^{2}$ Department of Mathematics, Pusan National University, Pusan 46241, Republic of Korea \\ Correspondence should be addressed to Ji-Hun Yoon; yssci99@pusan.ac.kr
}

Received 24 August 2019; Revised 23 December 2019; Accepted 26 February 2020; Published 26 March 2020

Guest Editor: Andriette Bekker

Copyright (C) 2020 Sun-Yong Choi and Ji-Hun Yoon. This is an open access article distributed under the Creative Commons Attribution License, which permits unrestricted use, distribution, and reproduction in any medium, provided the original work is properly cited.

\begin{abstract}
In this study, we model the returns of a stock index using various parametric distribution models. There are four indices used in this study: HSCEI, KOSPI 200, S\&P 500, and EURO STOXX 50. We applied 12 distributions to the data of these stock indices-Cauchy, Laplace, normal, Student's $t$, skew normal, skew Cauchy, skew Laplace, skew Student's $t$, hyperbolic, normal inverse Gaussian, variance gamma, and general hyperbolic-for the parametric distribution model. In order to choose the best-fit distribution for describing the stock index, we used the information criteria, goodness-of-fit test, and graphical tail test for each stock index. We estimated the value-at-risk (VaR), one of the most popular management concepts in the area of risk management, for the return of stock indices. Furthermore, we applied the parametric distributions to the risk analysis of equity-linked securities (ELS) as they are a very popular financial product on the Korean financial market. Relevant risk measures, such as VaR and conditional tail expectation, are calculated using various distributions. For calculating the risk measures, we used Monte Carlo simulations under the best-fit distribution. According to the empirical results, investing in ELS is more risky than investing in securities, and the risk measure of the ELS heavily depends on the type of security.
\end{abstract}

\section{Introduction}

The normal or Gaussian distribution is a widespread distribution for modeling in finance. However, in general, financial asset return distributions are not normal, which is one of the stylized facts of stock returns (see [1]). Much empirical research has shown that real data for stock price returns are generally characterized by skewness, kurtosis, and fat tails (see [2-4]). Therefore, multiple distributions have been used as an alternative to the normal one.

First, by adjusting the number of degrees of freedom, the Student's $t$ distribution has a fat tail compared to the normal distribution. The Student's $t$ distribution is used in financial engineering, such as option pricing and risk management (see [5-7]). Second, skew distributions allow us to take advantage of the skewness value. The skew normal (see [8-10]) and skew Student's $t$ (see [11-13]) are typical skew distributions. Third, a normal variance-mean mixture with generalized inverse Gaussian distribution can generate, for example, the generalized hyperbolic distribution introduced by Barndorff-Nielsen [14]. These types of distributions can be both symmetric and skewed, and their tails are heavier than those of the Gaussian distribution. The generalized hyperbolic distribution was used in many studies to fit a series of stock index returns (see [15-18]). Eberlein and Keller [19] and Eberlein [20], especially, demonstrated that a medium-tailed generalized hyperbolic family of distributions produces a more suitable fit to stock returns observed in the stock market. In addition, the variance gamma distribution is a subclass of the generalized hyperbolic distribution. Its skewness and kurtosis together describe the shape of the distribution. In the financial literature, Madan and Seneta [21] initially introduced the variance gamma distribution, and it has been used in various fields, for example, option pricing and synthetic CDO (collateralized debt obligations) pricing (see [22-24]). 
In this study, we utilized twelve parametric distributions-Cauchy, Laplace, normal, Student's $t$, skew normal, skew Cauchy, skew Laplace, skew Student's $t$, hyperbolic, normal inverse gamma, variance gamma, and generalized hyperbolic distributions - to fit the distribution of the stock index return. We also used four different stock indices: HSCEI, KOSPI 200, S\&P 500, and EURO STOXX 50. Furthermore, we applied the fitting results to the risk management because the return distribution plays an important role in risk measurement. These research approaches can be found in other studies (see $[2,12,13,25,26]$ ). Furthermore, Vernic [27], Bolance et al. [28], and Eling [29, 30] analyzed the skew normal and skew-student as favorable models for describing actuarial loss of data and the investment returns of insurance companies by fitting various parametric distributions. The difference from the previous studies is in the applications of various parametric distributions to the model risk of equity-linked securities (ELS). Although many financial products have been sold on the Korean market, we focus on ELS because it is one of the best-selling financial derivatives. ELS are a hybrid debt security whose investment return is connected to an underlying equity, such as stock indices-a group of stocks. ELS worth approximately 45.9 trillion won were issued in 2013 in Korea. Furthermore, risk management for ELS has become critical in the last several years. For example, only recently, the financial supervisor warned that sales of ELS tracking the Hang Seng China Enterprises Index (HSCEI) will be restricted as there is an increasing possibility that if such sales continue, it could trigger a knock-in option in most ELS products.

Since the Basel Committee on Banking Supervision introduced the value-at-risk (VaR) in 1996, it has been widely used as a risk measure in the risk management industry. VaR specifies the maximum amount an investment may lose, within a given probability, in a specified period of time. It is well known that $\mathrm{VaR}$ provides several benefits in terms of risk management. Above all, it captures an important aspect of risk in a single number, and it is useful to compare different assets and portfolios (see [31, 32]).

Measuring risk is crucial to control the risk and expected losses for both banks and companies. In addition, $\mathrm{Hu}$ and Kercheval [33] said that the choice of risk measure is less important for portfolio management than the choice of distribution family. In other words, the best choice of return distribution is a critical issue for portfolio management. However, there are two typical measures for risk, VaR (see [34]) and conditional tail expectation (CTE) (see [35]). They both have some pros and cons. For example, in the case of value-at-risk (VaR), it is easy to understand the VaR figures, but those figures are volatile. If we refer to CTE, it has subadditivity (the portfolio effect) and it is less volatile than the $\mathrm{VaR}$. The use of risk measures - VaR and CTE-is required by the Basel Committee on Banking Supervision in determining a bank's risk profile.

There are two different approaches to the estimation of VaR: the nonparametric method and the fully parametric method. A short review of these methods is as follows. Historical simulation is one of the most commonly used nonparametric approaches, and it is the easiest way to estimate $\mathrm{VaR}$. One of the most popular parametric models is the delta-normal model. This model assumes that the return of a portfolio follows a normal distribution. However, this assumption is not supported by results from the real market; it has been shown that many financial assets have return distributions with "fatter tails" than present in the normal distribution (i.e., values that are further from the mean have higher probabilities than a normal distribution would suggest). In consequence, a great deal of effort has been put into developing parametric models to describe the fat-tailed distribution.

In this study, we use VaR and CTE in order to measure the risk of a stock index and financial derivative. Thus, we estimate $\mathrm{VaR}$ for the return of stock indices and evaluate the $\mathrm{VaR}$ backtest. In order to measure the risk of ELS, we implement several steps as follows: first, we investigate whether these models are appropriate for describing the stock indices - that is, we fit the return to the parametric distributions and find the best-fit distribution for each stock index. Second, we calculate VaR and CTE for a single stock index, portfolios consisting of stock indices and ELS, using the historical data. Finally, we use the Monte Carlo method to simulate VaR and CTE for the ELS.

The rest of this paper is as follows. In Section 2, we briefly review various parametric distributions. Then, we demonstrate the fitting results and determine the best-fit distributions via information criteria, goodness-of-fit test, and graphical tail test for each stock index in Section 3. Section 4 gives the application results for the stock indices and ELS. Section 5 concludes the paper.

\section{Distributions}

As mentioned previously, we used 12 parametric distributions in this study: Cauchy, Laplace, normal, Student's $t$, skew normal, skew Cauchy, skew Laplace, skew Student's $t$, hyperbolic, normal inverse Gaussian, variance gamma, and general hyperbolic. The Cauchy distribution is named after the mathematician A.L. Cauchy (1789-1857). By using location $\mu$ and scale $(\sigma>0)$ as parameters, the probability density function (pdf) of the Cauchy distribution is

$$
f(x ; \mu, \sigma)=\frac{1}{\pi} \frac{\sigma}{\sigma^{2}+(x-\mu)^{2}}, \quad x \in \mathbb{R} .
$$

The random variable $X$ has the Laplace distribution location $\mu$ and scale $\sigma(>0)$ parameters if it has the following pdf:

$$
f(x ; \mu, \sigma)=\frac{1}{2 \sigma} \exp \left(-\frac{|x-\mu|}{\sigma}\right), \quad x \in \mathbb{R} .
$$

A normal distribution is defined by two parameters: mean (or location) $\mu$ and variance (or scale) $\sigma(>0)$. The pdf of the normal distribution is

$$
f(x ; \mu, \sigma)=\frac{1}{\sigma \sqrt{2 \pi}} \exp \left(-\frac{(x-\mu)^{2}}{2 \sigma^{2}}\right), \quad x \in \mathbb{R} .
$$

The Student's $t$ distribution has heavier tails than a normal distribution and the degrees of freedom $(\nu \geq 1)$ 
controls the heavy-tailedness. Student's $t$ distribution can be described by a normal and a Gamma distribution. Let $Y$ be a random variable following the normal distribution with zero-mean and the scale $\sigma$. Let $U$ have a Gamma distribution $\operatorname{Gam}((\nu / 2),(\nu / 2))$ in shape/rate parameterization (see [36]). Then, the random variable

$$
X=\mu+\frac{Y}{\sqrt{U}}
$$

admits the Student's $t$ distribution with mean $\mu$, scale $\sigma$, and degrees of freedom parameter $v$ (see [37]). Its pdf is

$$
f(x ; \mu, \sigma, \nu)=\frac{\Gamma(\nu+1 / 2)}{\sqrt{\nu \pi} \sigma \Gamma(\nu / 2)}\left(1+\frac{1}{\nu}\left(\frac{x-\mu}{\sigma}\right)^{2}\right)^{-(\nu+1 / 2)}, \quad x \in \mathbb{R},
$$

where $\Gamma$ is the gamma function. Although the pdf of Student's $t$ can be generated by chi-square distribution, this distribution is the marginal posterior distribution for the normal mean with unknown variance and conjugate prior distribution (see [38]). Thus, three-parameter Student's $t$ distribution (5) is naturally used in many Bayesian inference problems.

Figure 1 illustrates the pdf of the Cauchy, Laplace, standard normal, and Student's $t$ distributions with different degrees of freedom. The pdf of Cauchy, Laplace, and Student's $t$ distribution has a fat tail compared to the normal distribution. Furthermore, the pdf of Student's $t$ converges to a normal distribution when the number of degrees of freedom $v$ tends to infinity. The case of $v=1$ is the Cauchy distribution.

The Cauchy, Laplace, normal, and Student's $t$ distribution cannot show the asymmetry of the pdf. Therefore, as an extension to the normal distribution, in order to accommodate asymmetry, the skew normal distribution was first suggested by Azzalini [8]. Let $\phi(\cdot)$ and $\Phi(\cdot)$ be the standard normal density function and the cumulative density function (cdf), respectively. A random variable $X$ is said to have a skew normal distribution with the skew parameter $\alpha \in \mathbb{R}$. The pdf of the skew normal distribution is then given as

$$
f(x ; \alpha)=2 \phi(x) \Phi(\alpha x), \quad x \in \mathbb{R} .
$$

Location and scale parameters can be included via the linear transformation $Y=\mu+\sigma X$, which follows the skew normal distribution with location $\mu$, scale $\sigma$, and shape parameter $\alpha \in \mathbb{R}$ (denoted by $\left.S N\left(\mu, \sigma^{2}, \alpha\right)\right)$. The skew normal distribution has a kurtosis that is a little bit higher than the normal distribution, which is one of its limitations.

Similar to Azzalini's study [8], Gupta and Huang [39] and Gupta et al. [40] introduced various skew distributions. One of them is the skew Cauchy distribution. The pdf of the skew Cauchy distribution is

$$
f(x ; \mu, \sigma, \alpha)=\frac{\sigma[1+2(\arctan (\alpha x / \sigma) / \pi)]}{\pi\left(\sigma^{2}+x^{2}\right)}, \quad x \in \mathbb{R} .
$$

Another technique of importing skewness into a symmetric distribution was developed by Fernández and Steel [41]. The idea was to convert a symmetric pdf into a skewed one by postulating inverse scale factors in the positive and

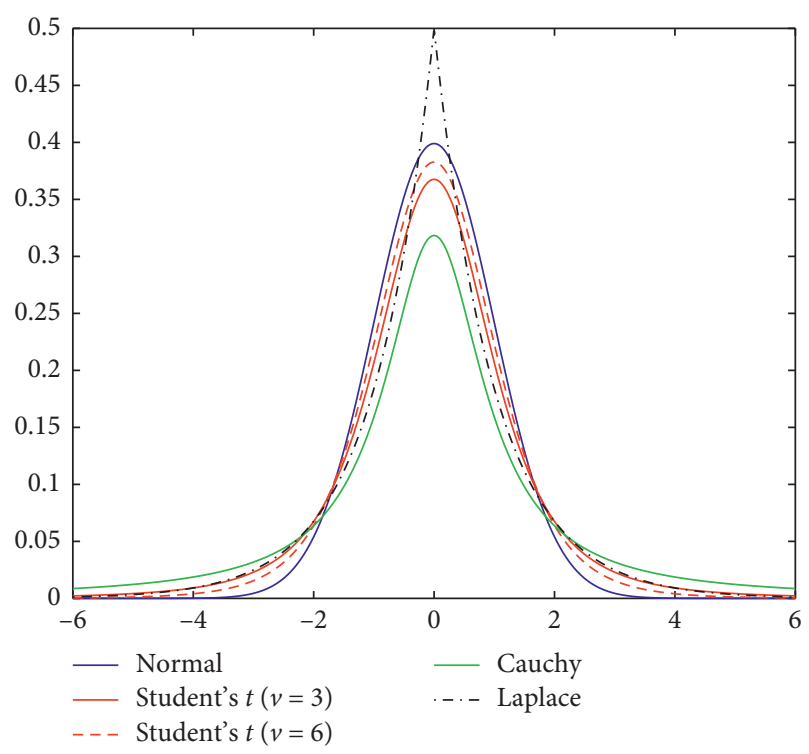

Figure 1: Cauchy, Laplace, normal, and Student's $t$ distributions with the parameters $\mu=0, \sigma=1$, and $\nu=3,6$.

negative orthants. Based on this technique, the pdf of an asymmetric Laplace distribution is given by

$$
f(x ; \mu, \sigma, \alpha)=\frac{1}{\sigma} \frac{\alpha}{1+\alpha^{2}}\left\{\begin{array}{ll}
\exp \left(-\frac{\alpha}{\sigma}(x-\mu)\right), & \text { for } x \geq \mu, \\
\exp \left(\frac{1}{\alpha \sigma}(x-\mu)\right), & \text { for } x<\mu,
\end{array} \quad x \in \mathbb{R},\right.
$$

where $\mu, \sigma(>0)$, and $\alpha(>0)$ are the location, scale, and skewness parameters, respectively. Figure 2 displays the pdf of skew Cauchy and skew Laplace distributions with varying $\alpha$ values. Through these figures, we can observe the asymmetric distributions.

According to Nurminen et al. [42], the univariate skew Student's $t$ distribution is parameterized by the location $\mu$, scale $\sigma$, skew $\alpha$, and degrees of freedom $\nu$. The pdf of skew Student's $t$ distribution is

$$
f(x ; \mu, \sigma, \alpha, \nu)=2 t\left(x ; \mu, \sigma^{2}+\alpha^{2}, \nu\right) T(\bar{x} ; 0,1, \nu+1), \quad x \in \mathbb{R},
$$

where $t(x ; \mu, \sigma, \nu)$ is the pdf of Student's $t$ distribution given by $(5)$ and $\bar{x}=((x-\mu) \alpha / \sigma)\left(\nu+1 / \nu\left(\sigma^{2}+\alpha^{2}\right)+(x-\mu)^{2}\right)^{1 / 2}$. Also, $T(\cdot ; 0,1, \nu)$ is the cdf of Student's $t$ distribution with degrees of freedom $\nu$.

In Figure 3, the skew normal and skew Student's $t$ distributions with the skew parameter $\alpha=0$ are equivalent to the normal and Student's $t$, respectively. Furthermore, according to the level of the skew parameter of the distribution, we can have positive or negative skewed pdf in the skew normal and skew Student's $t$ distributions.

The general hyperbolic distribution (ghyp) was introduced by Barndorff-Nielsen [43], and the ghyp can be parameterized in several ways. Following Prause et al. [44], the pdf of the ghyp is 


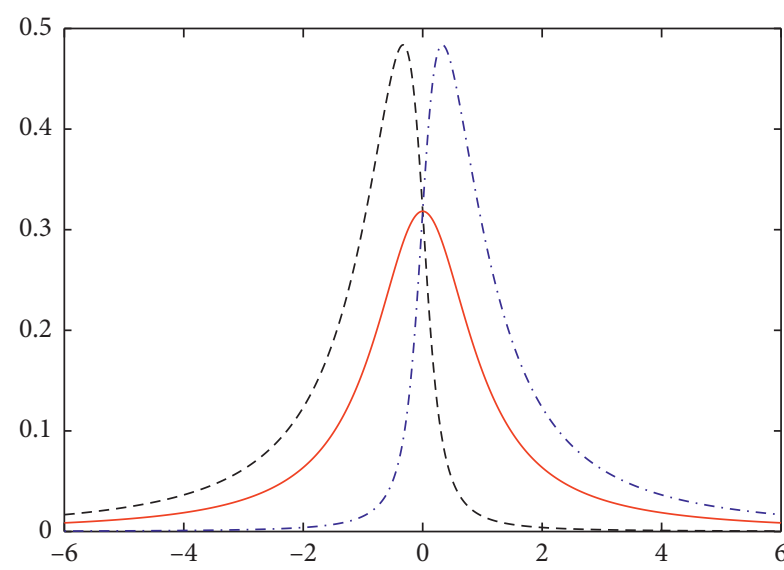

- - Skew Cauchy $(\alpha=-3)$

- Skew Cauchy $(\alpha=0)$

... Skew Cauchy $(\alpha=3)$

(a)

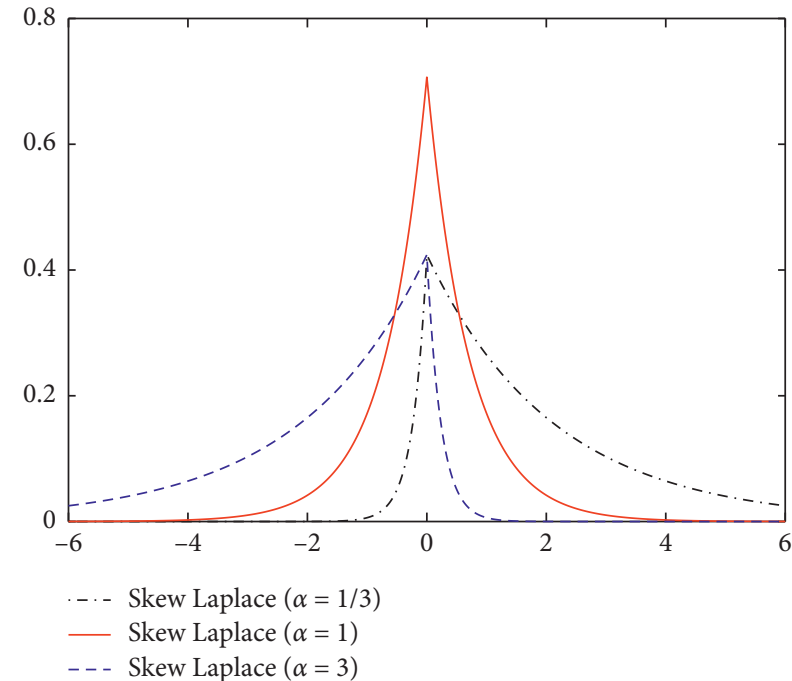

(b)

Figure 2: The pdf of skew Cauchy and skew Laplace distributions with different parameters of $\alpha$. (a) Parameters $\mu=0 ; \sigma=1$. (b) Parameters $\mu=0 ; \sigma=1$.

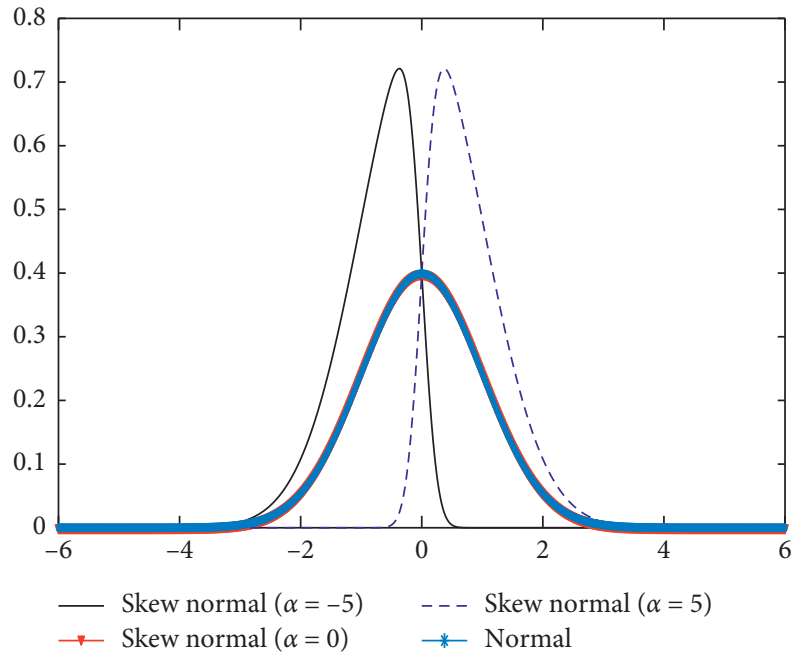

(a)

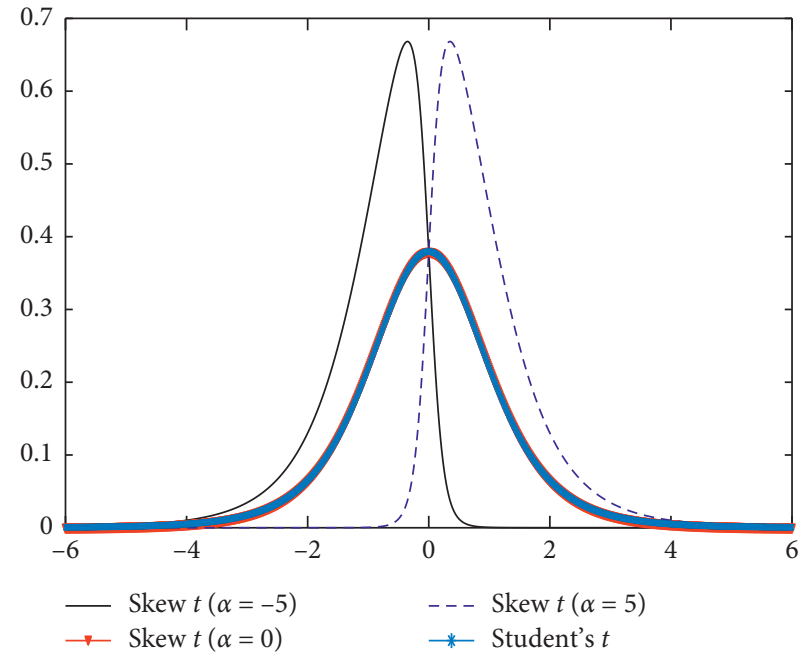

(b)

Figure 3: Skew normal distribution and skew Student's $t$ distribution with the skew parameter $\alpha=-5,0,5$. (a) Parameters $\mu=0 ; \sigma=1 ; \nu=5$. (b) Parameters $\mu=0 ; \sigma=1$.

$$
f(x ; \lambda, \mu, \sigma, \alpha, \beta)=\frac{\left(\beta^{2}-\alpha^{2}\right) K_{\lambda-1 / 2}\left(\beta \sqrt{\sigma^{2}+(x-\mu)^{2}}\right) \exp (\alpha(x-\mu))}{\sqrt{2 \pi} \beta^{\lambda-1 / 2} \sigma^{\lambda} K_{\lambda}\left(\sigma \sqrt{\beta^{2}-\alpha^{2}}\right)\left(\sqrt{\sigma^{2}-(x-\mu)^{2}}\right)^{1 / 2-\lambda}},
$$

where $K_{\lambda}$ denotes a modified Bessel function of the third kind with index $\lambda$ (see [45]).

$$
\begin{array}{ll}
\sigma \geq 0,|\alpha|<\beta, & \text { if } \lambda>0, \\
\sigma>0,|\alpha|<\beta, & \text { if } \lambda=0, \\
\sigma>0,|\alpha| \leq \beta, & \text { if } \lambda<0 .
\end{array}
$$

A remarkable aspect is that ghyp distributions envelop many special cases and limiting distributions, such as, for example, the Student's $t$, normal, hyperbolic, and normal inverse Gaussian distributions (see [19]). We describe some of the special cases.

For $\lambda=1$, we get the hyperbolic distribution. This type of distribution is also generated by the normal and generalized 
inverse Gaussian distributions. The hyperbolic distribution decreases exponentially, which is more slow than the normal distribution. The pdf of the hyperbolic distribution is

$$
\begin{aligned}
f(x ; \mu, \sigma, \alpha, \beta)= & \frac{\sqrt{\beta^{2}-\alpha^{2}}}{2 \beta \sigma K_{1}\left(\sigma \sqrt{\beta^{2}-\alpha^{2}}\right)} \\
& \cdot \exp \left(-\beta \sqrt{\sigma^{2}+(x-\mu)^{2}}+\alpha(x-\mu)\right), \quad x \in \mathbb{R},
\end{aligned}
$$

where $\beta$ is responsible for the steepness. The other $\mu, \sigma$, and $\alpha$ are the location, scale, and skewness parameters, respectively.

We obtain the normal inverse Gaussian (NIG) distribution by letting $\lambda=-(1 / 2)$ in (11). The pdf of NIG is

$$
f(x ; \mu, \sigma, \alpha, \beta)=\frac{\sigma \beta \exp \left(\sigma \sqrt{\beta^{2}-\alpha^{2}}\right) K_{1}\left(\beta \sqrt{\sigma^{2}+(x-\mu)^{2}}\right) \exp (\alpha(x-\mu))}{\pi \sqrt{\sigma^{2}+(x-\mu)^{2}}}, \quad x \in \mathbb{R},
$$

where $\sigma>0$ and $0<|\alpha|<\beta$.

On the contrary, the variance gamma distribution is also described by the normal and gamma distributions, and the tails of the variance gamma distribution decrease more slowly than the normal distribution. The pdf of variance gamma is

$$
\begin{aligned}
f(x ; \mu, \sigma, \alpha, \lambda)= & \sqrt{\frac{\pi}{2}} \frac{\lambda^{1 / \lambda} \exp \left((\alpha(x-\mu)) / \sigma^{2}\right)}{\sigma \Gamma(1 / \lambda)} \\
& \cdot\left(\frac{|x-\mu|}{\sqrt{\alpha^{2}+\left(2 \sigma^{2} / \lambda\right)}}\right)^{1 / \lambda-1 / 2} \\
& \cdot K_{1 / \lambda-1 / 2}\left(\frac{[x-\mu) \sqrt{\alpha^{2}+\left(2 \sigma^{2} / \lambda\right)}}{\sigma^{2}}\right), \quad x \in \mathbb{R},
\end{aligned}
$$

where $\mu, \sigma, \alpha$, and $\lambda(>0)$ are the location, scale, skewness, and shape parameters, respectively.

Figures 4-7 show the pdf of the hyp, NIG, ghyp, and variance gamma distributions, respectively. The plots can show a skewed and high kurtosis distribution by adjusting the skew parameter $(\alpha)$ and the kurtosis parameter $(\beta, \lambda)$.

Table 1 summarizes the features of each distribution. These features are normality, kurtosis, and skewness, and they can be controlled by the skew Student's $t$, hyperbolic, NIG, variance gamma, and generalized hyperbolic distributions.

\section{Fitting and Selecting}

3.1. Fitting Distribution. The four stock indices are considered for the period January 2013 to December 2017, amounting to 5 years of business days. Figure 8 illustrates the time series of price, log return, and histogram for each index. Furthermore, Table 2 describes basic statistics for the stock indices. By reading the skewness values presented in Table 2, we can observe that the return distributions for KOSPI 200 and S\&P 500 show negative skewness. All return distributions are more fat-tailed relative to the normal distribution.
In addition, Jarque-Bera statistics for all return series indicate that the four stock indices have nonnormal return distributions.

We can estimate the parameters of the distributions via maximum likelihood, and all models are implemented in the $R$ packages fGarch, ghyp, sn, ald, VGAM, and MASS. In addition, the approach assumes that the time series is approximately independent and identically distributed. Hence, we plotted the autocorrelation function (ACF) of the log return series and squared the log return series for each stock index in Figure 9. Figure 9 indicates that while the log return series are serially uncorrelated, the squared log return series are serially correlated, which is in accordance with the stylized facts introduced by Cont [1]. In this study, we utilize the GARCH $(1,1)$ model in order to remove the dependence in return series according to the arguments made by McNeil et al. [46]. We further present the definition of the GARCH $(1,1)$ model.

Definition 1 (GARCH $(1,1))$. The process $\left(X_{t}\right)$ is GARCH $(1,1)$ if it is covariance stationary and satisfies the following equations:

$$
\begin{aligned}
X_{t} & =\sigma_{t} Z_{t}, \\
\sigma_{t}^{2} & =\alpha_{0}+\alpha_{1} X_{t-1}^{2}+\beta_{1} \sigma_{t-1}^{2},
\end{aligned}
$$

where $\alpha_{0}>0, \alpha_{1} \geq 0, \beta_{1} \geq 0$, and $\alpha_{1}+\beta_{1}<1 ; z_{t}$ is a sequence of random variables following independent and identical distribution with zero mean and unit variance.

We create a filtered return series by subtracting the mean $\mu$ of the raw return series and then calibrating the GARCH $(1,1)$ parameters $\left(\alpha_{0}, \alpha_{1}\right.$, and $\left.\beta_{1}\right)$. We then obtain the filtered return series defined as follows:

$$
\widehat{X_{t}}=\frac{X_{t}-\mu}{\sigma_{t}}
$$

$\widehat{X_{t}}$ should be approximately independent and identically distributed (i.i.d). 


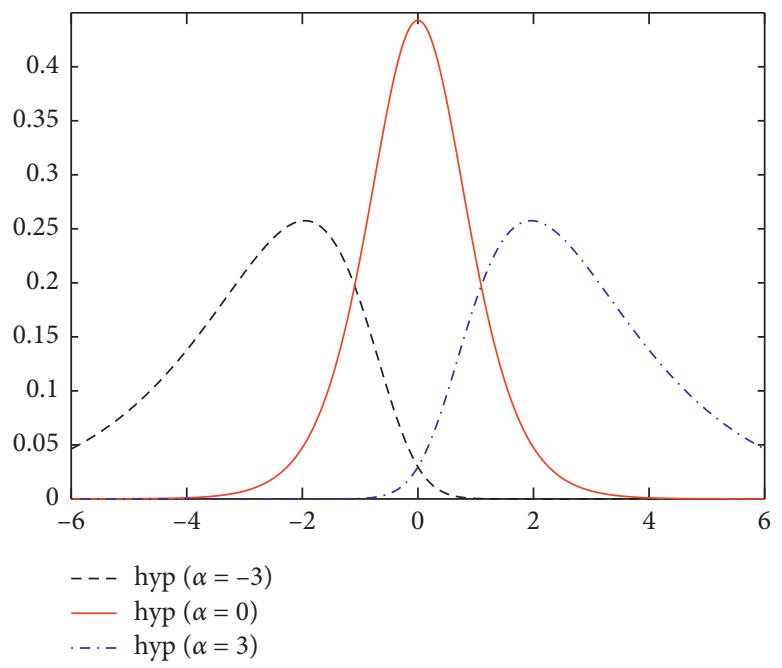

(a)

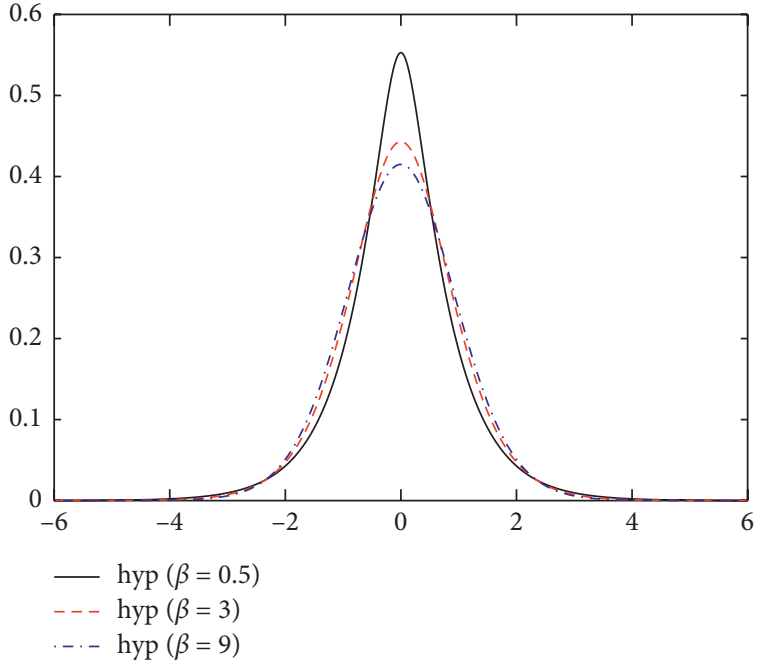

(b)

Figure 4: The pdf of the hyperbolic distributions with different parameters $\alpha$ and $\beta$. (a) Parameters $\mu=0 ; \sigma=1 ; \beta=3$. (b) Parameters $\mu=0$; $\sigma=1 ; \alpha=0$.

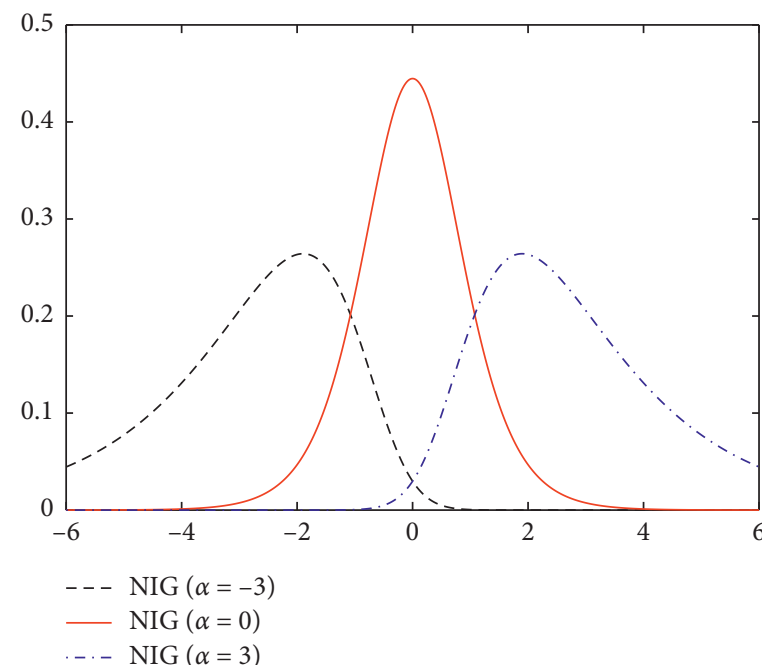

(a)

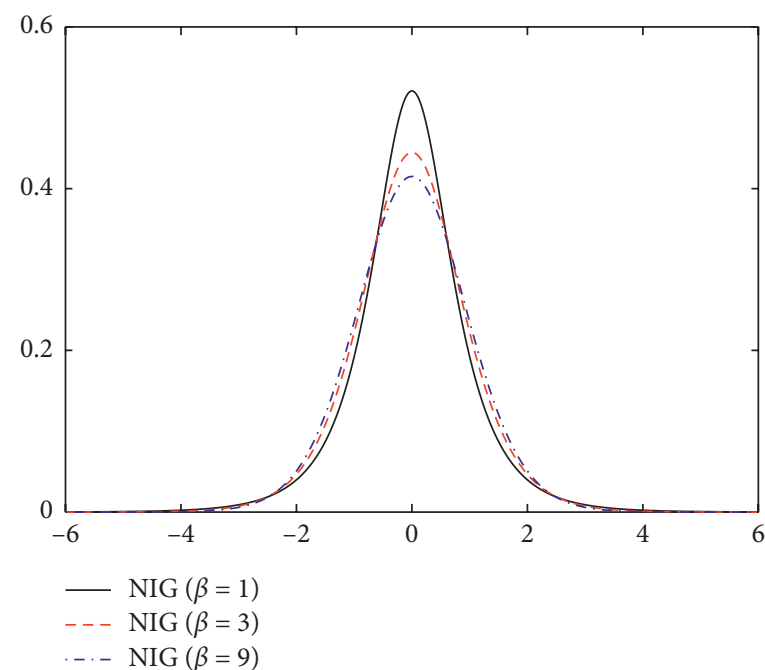

(b)

Figure 5: The pdf of the NIG with different parameters $\alpha$ and $\beta$. (a) Parameters $\mu=0 ; \sigma=1 ; \beta=3$. (b) Parameters $\mu=0$; $\sigma=1 ; \alpha=0$.

The ACF of both filtered return series and squared filtered return series for each stock index is illustrated in Figure 10. Figure 10 shows that there is no dependence in both filtered return series and squared return series. Therefore, now we can apply the maximum likelihood method to the filtered return series of the four stock indices. The estimation results of the 12 distributions for each index are given in Tables 3-6 in Appendix.

The next step is to compare the distribution using several tools. In this study, we chose three tools to find the best-fit distribution for each stock index. The first such tool is the information criteria-Akaike information criterion and Bayesian information criterion. Following are the definitions of the information criteria.
Definition 2 (Akaike information criterion [47]). Akaike information criterion (AIC) is a measure of goodness of fit defined as

$$
\mathrm{AIC}=2 \cdot k-2 \log \mathscr{L}(\widehat{\theta}),
$$

where $k$ is the number of parameters to be estimated in the model, $\widehat{\theta}$ are the estimated parameters that maximize the likelihood (or log-likelihood), and $\log \mathscr{L}(\widehat{\theta})$ is the maximum value of the log-likelihood.

Definition 3 (Bayesian information criterion [48]). Bayesian information criterion (BIC) or Schwarz criterion is a measure of goodness of fit defined as 


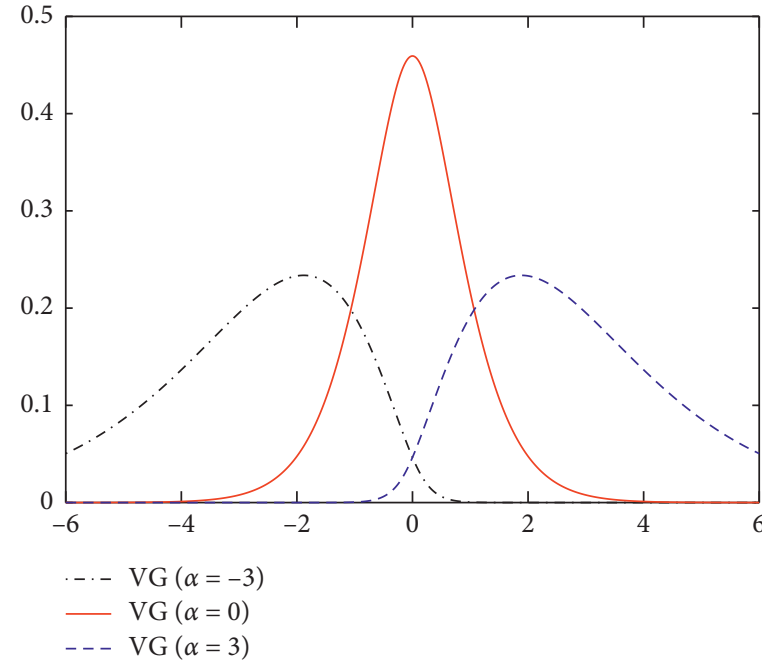

(a)

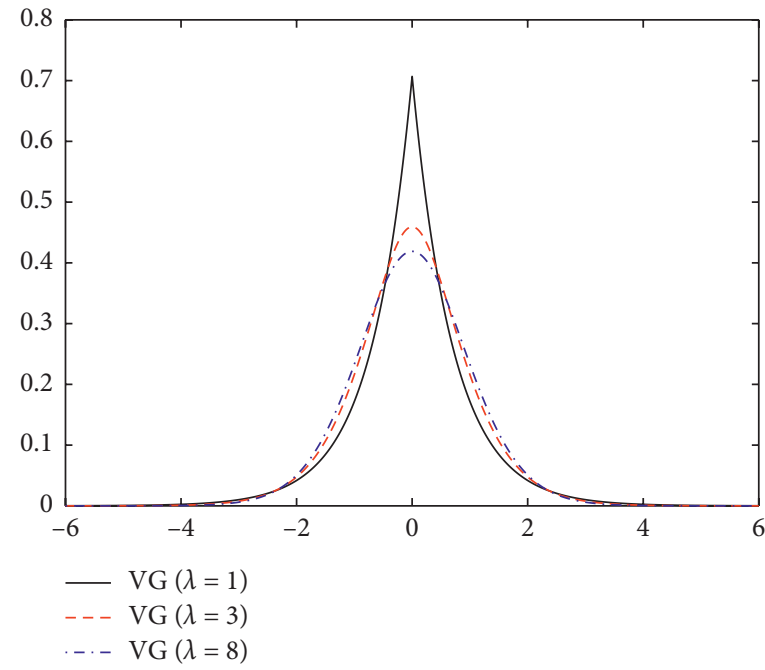

(b)

Figure 6: The pdf of the variance gamma distributions with different parameters $\alpha$ and $\lambda$. (a) Parameters $\mu=0 ; \sigma=1 ; \beta=3$. (b) Parameters $\mu=0 ; \sigma=1 ; \alpha=0$.

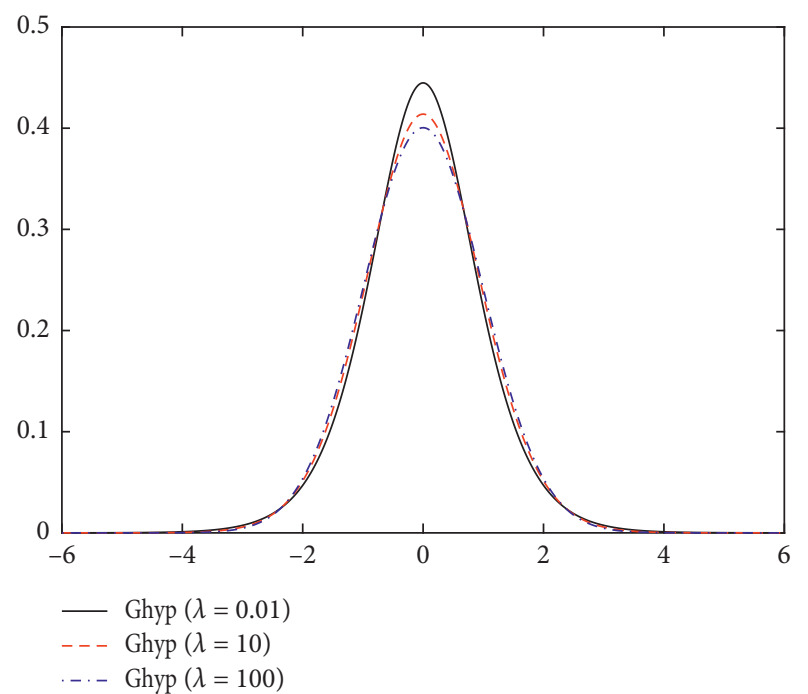

Figure 7: The pdf of the ghyp with different parameters $\lambda$. Parameters $\mu=0 ; \sigma=1 ; \alpha=0 ; \beta=3$.

TABLE 1: The features of distributions.

\begin{tabular}{lccc}
\hline$[1.2 \mathrm{pt}]$ & Normality & Kurtosis & Skewness \\
\hline Cauchy & $\mathrm{X}$ & $\mathrm{O}$ & $\mathrm{X}$ \\
Laplace & $\mathrm{X}$ & $\mathrm{O}$ & $\mathrm{X}$ \\
Normal & $\mathrm{O}$ & $\mathrm{X}$ & $\mathrm{X}$ \\
Student's $t$ & $\mathrm{O}$ & $\mathrm{O}$ & $\mathrm{X}$ \\
Skew normal & $\mathrm{O}$ & $\mathrm{X}$ & $\mathrm{O}$ \\
Skew Cauchy & $\mathrm{O}$ & $\mathrm{X}$ & $\mathrm{O}$ \\
Skew Laplace & $\mathrm{O}$ & $\mathrm{X}$ & $\mathrm{O}$ \\
Skew Student's $t$ & $\mathrm{O}$ & $\mathrm{O}$ & $\mathrm{O}$ \\
Hyperbolic & $\mathrm{O}$ & $\mathrm{O}$ & $\mathrm{O}$ \\
NIG & $\mathrm{O}$ & $\mathrm{O}$ & $\mathrm{O}$ \\
Variance gamma & $\mathrm{O}$ & $\mathrm{O}$ & $\mathrm{O}$ \\
G. hyperbolic & $\mathrm{O}$ & $\mathrm{O}$ & $\mathrm{O}$ \\
\hline
\end{tabular}

$$
\mathrm{BIC}=\log (n) \cdot k-2 \log \mathscr{L}(\widehat{\theta}),
$$

where $n$ is the number of observations and $\widehat{\theta}$ are the estimated parameters that maximize the likelihood (or loglikelihood).

The AIC and BIC can be used to compare models based on different probability distributions. In the model selection application, the optimally fitted model is identified by the minimum value of AIC and BIC. We chose the fitted candidate distribution corresponding to the minimum value of AIC and BIC. This means that, for each index, we chose the best-fit distribution among the 12 distributions based on the AIC and BIC values given in Table 7.

The preferred distribution is the one with the lowest AIC and BIC values. Fortunately, for each stock index, the distribution has the lowest $\mathrm{AIC}$ and an equivalent BIC. Therefore, we chose the best-fit distribution for each stock index using the information criteria. Consequently, the bestfit distributions are Student's $t$ for HSCEI and variance gamma for KOSPI 200, S\&P 500, and EURO STOXX 50.

3.2. Goodness-of-Fit Test. In order to enhance the robustness of the fitting results, we additionally performed the Kolmogorov-Smirnov test for the goodness-of-fit test. According to the test results given in Table 8, we can determine which theoretical distribution differs significantly from the given return distribution for each stock index. Based on the Kolmogorov-Smirnov test, we determined that the distributions in bold are not able to describe the return distribution with the given significance level of $5 \%$.

The Kolmogorov-Smirnov test uses the whole samples to calculate the statistics, which represent the maximum difference value between the empirical distribution function and the theoretical distribution function. However, in extreme cases, the left and the right tails of the return distribution are usually affected in terms of risk management. In other words, the tails of the return distribution and the 


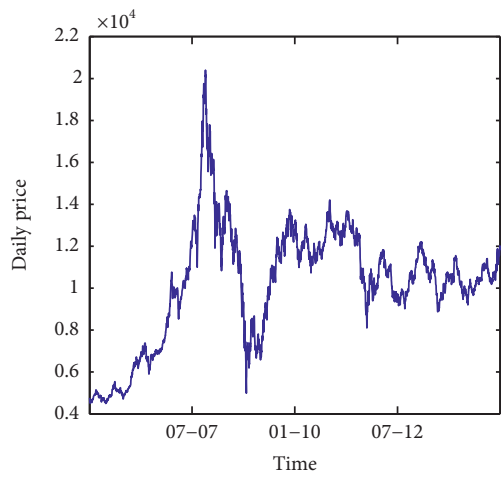

(a)

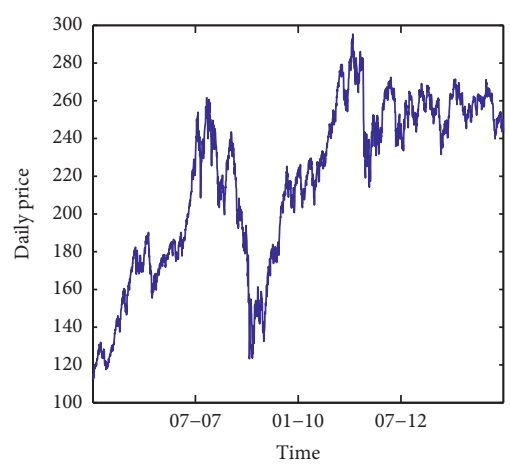

(d)

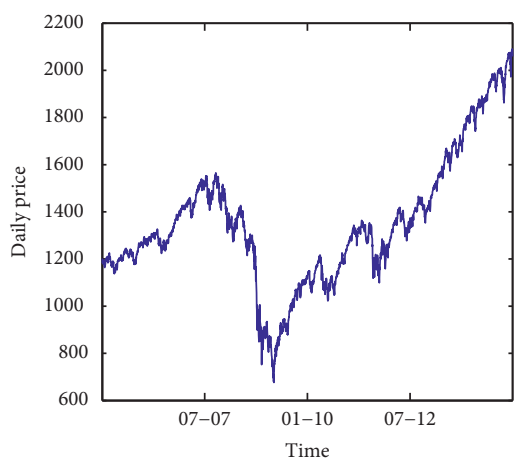

(g)

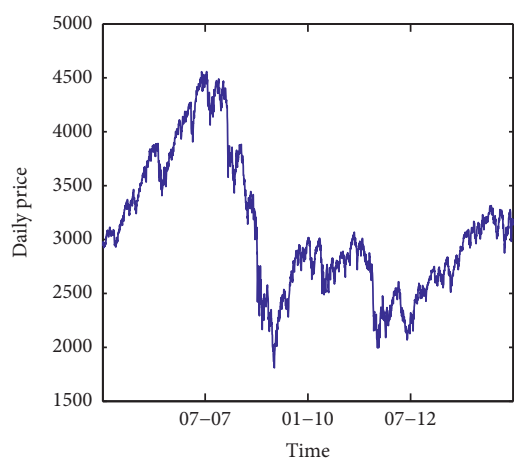

(j)

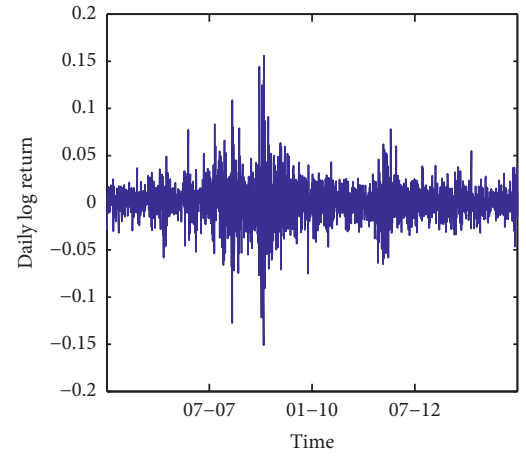

(b)

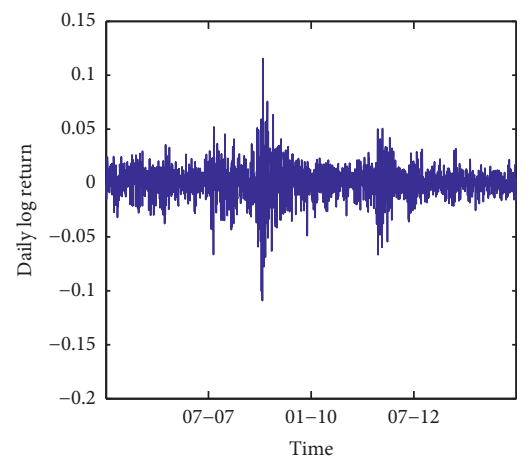

(e)

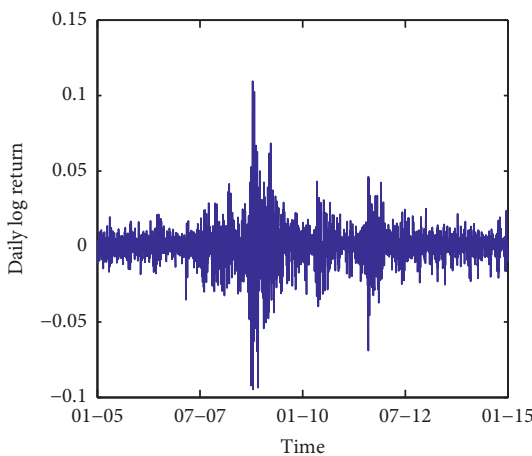

(h)

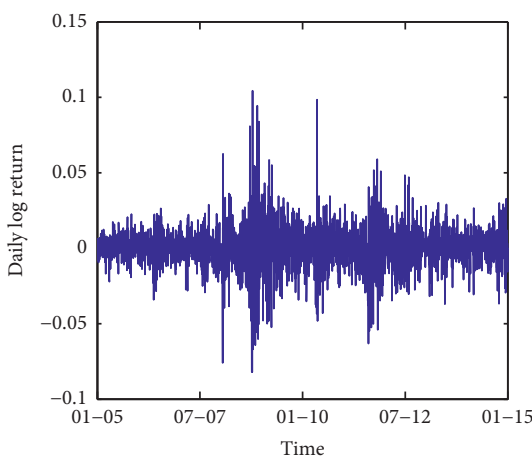

(k)

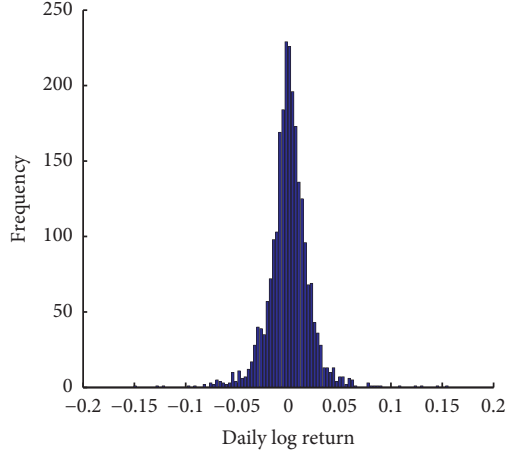

(c)

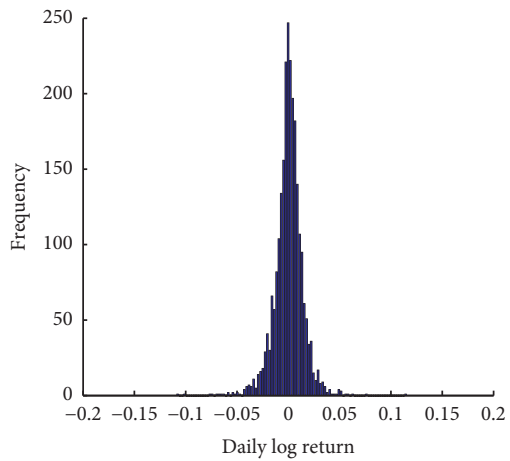

(f)

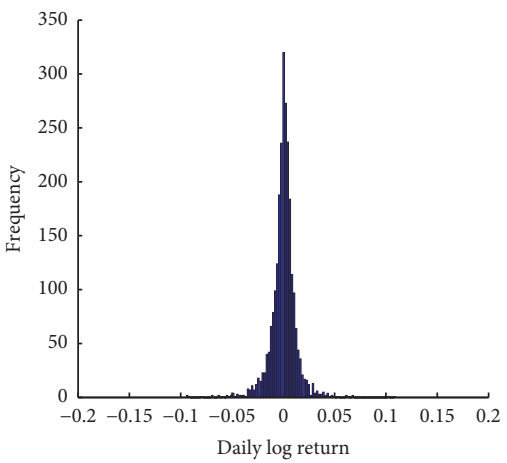

(i)

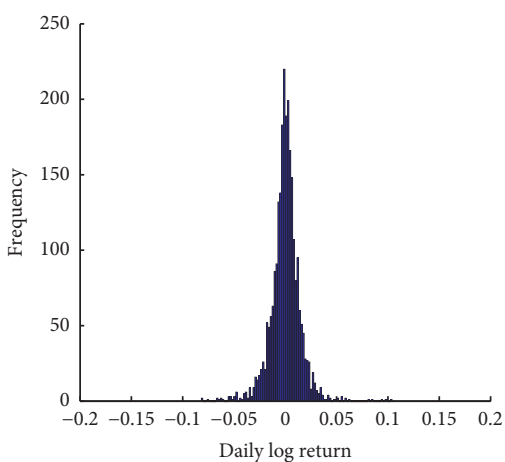

(l)

Figure 8: Time series of price, return, and histogram for the four indices. (a) HSCEI index prices. (b) Logarithmic time series of HSCEI returns. (c) Histogram of HSCEI returns. (d) KOSPI 200 index prices. (e) Logarithmic time series of KOSPI 200 returns. (f) Histogram of KOSPI 200 returns. (g) S\&P 500 index prices. (h) Logarithmic times series of S\&P 500 returns. (i) Histogram of S\&P 500 returns. (j) EURO STOXX 50 index prices. (k) Logarithmic time series of EURO STOXX 50 returns. (l) Histogram of EURO STOXX 50 returns. 
TABle 2: Summary statistics for the four stock indices. ${ }^{*}$ The minimum value $(<0.001)$ of $p$ value in the Jarque-Bera test.

\begin{tabular}{lcccc}
\hline & HSCEI & KOSPI 200 & S\&P 500 & EURO STOXX 50 \\
\hline Obs. & 2439 & 2481 & 2516 & 2555 \\
Mean & $3.7829 e-4$ & $3.0202 e-4$ & $2.1388 e-4$ & $2.2583 e-4$ \\
Std. dev. & 0.0207 & 0.0141 & 0.0129 & 0.01441 \\
Skewness & 0.0411 & -0.4216 & -0.3339 & 0.0238 \\
Kurtosis & 10.3129 & 10.0186 & 14.0144 & 9.3072 \\
Jarque-Bera & $543.54^{*}$ & $516.58^{*}$ & $1276.5^{*}$ & $423.52^{*}$ \\
\hline
\end{tabular}

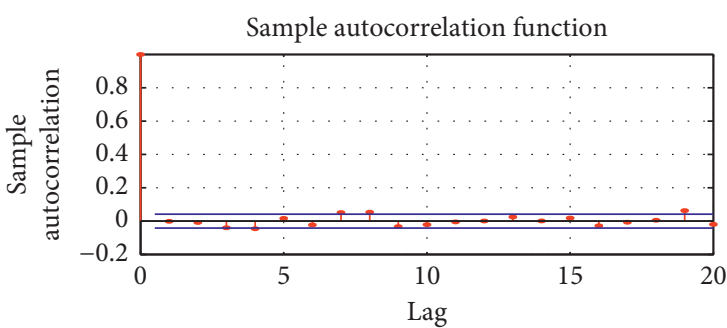

(a)

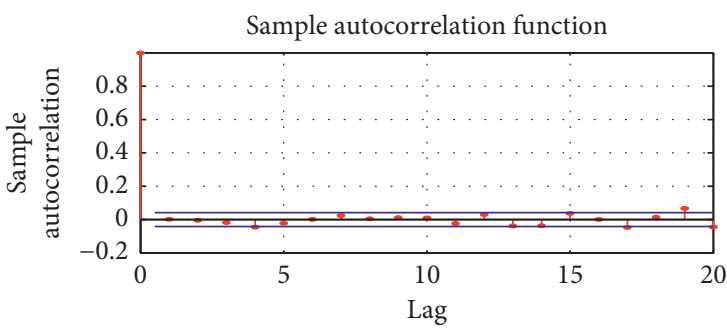

(c)

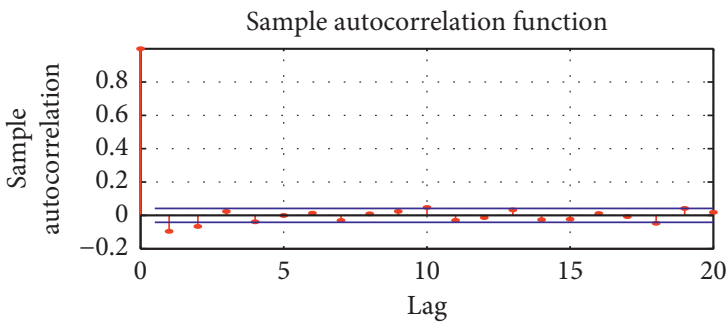

(e)

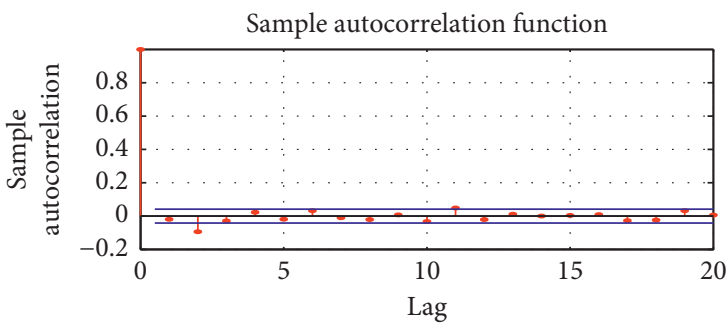

(g)

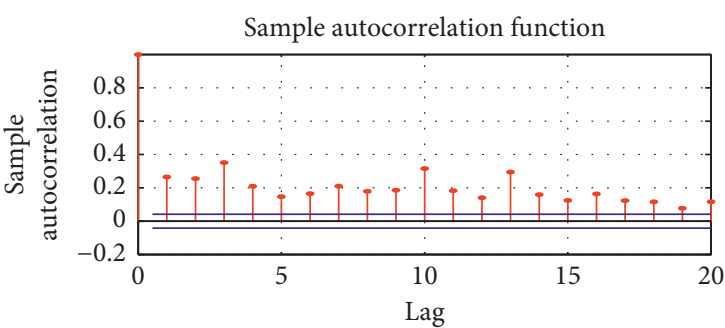

(b)

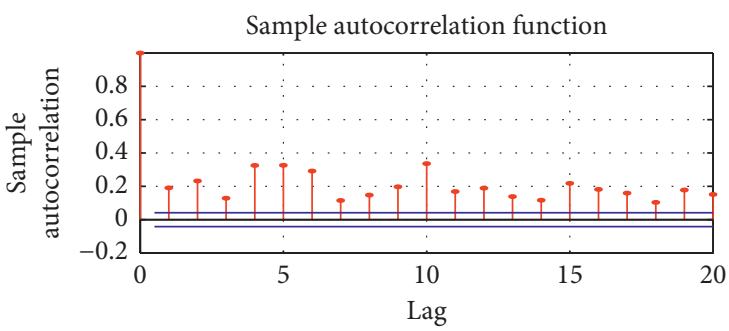

(d)

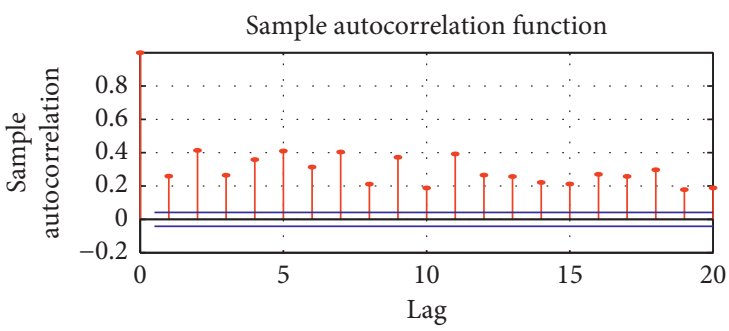

(f)

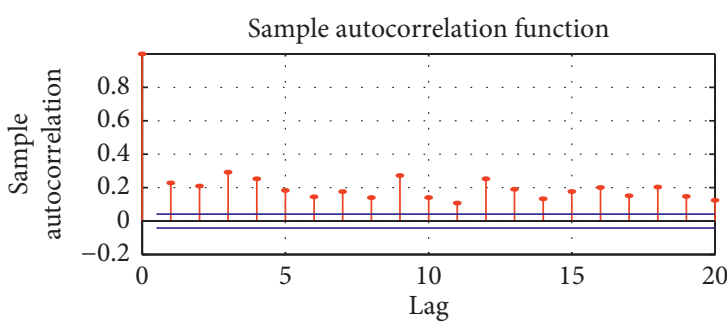

(h)

FIGURE 9: Correlograms for the four-stock index log return series. (a) ACF of return for HSCEI. (b) ACF of squared return for HSCEI. (c) ACF of return for KOSPI 200. (d) ACF of squared return for KOSPI 200. (e) ACF of return for S\&P 500. (f) ACF of squared return for S\&P 500. (g) ACF of return for EURO STOXX 50. (h) ACF of squared return for EURO STOXX 50.

risk management are interrelated. Therefore, we used a graphical left tail test for examining the fit in the tails. The graphical tests were performed as follows (see [49]): (i) Let $\widehat{F}(x)$ denote the estimated cdf of the fitted distribution and $\left(X_{(1)}, \ldots, X_{(N)}\right)$ the order statistic of the historical data 


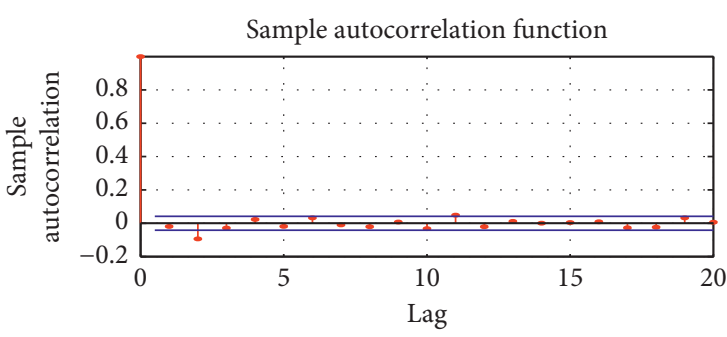

(a)

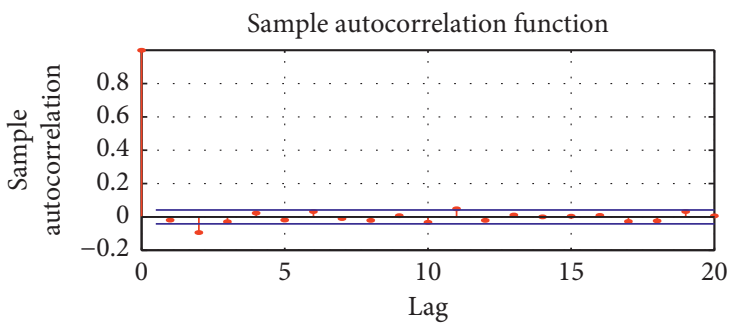

(c)

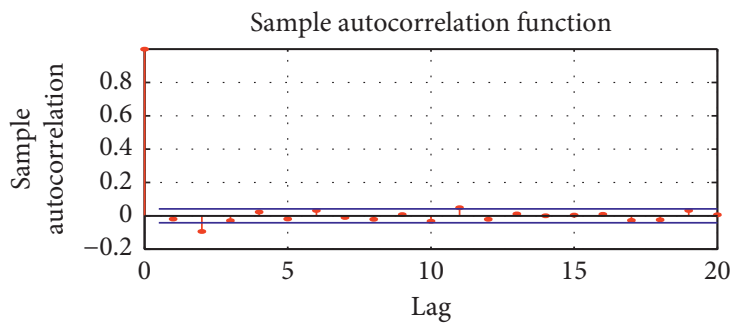

(e)

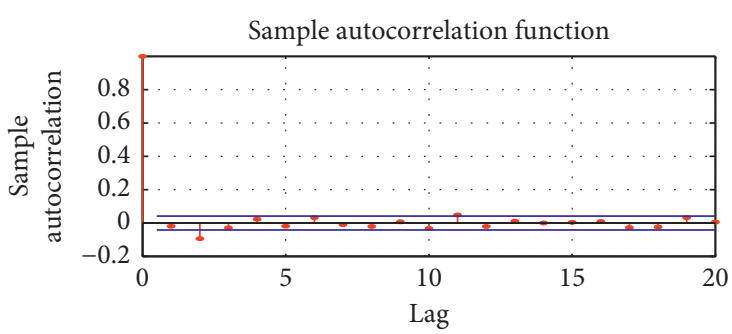

(g)

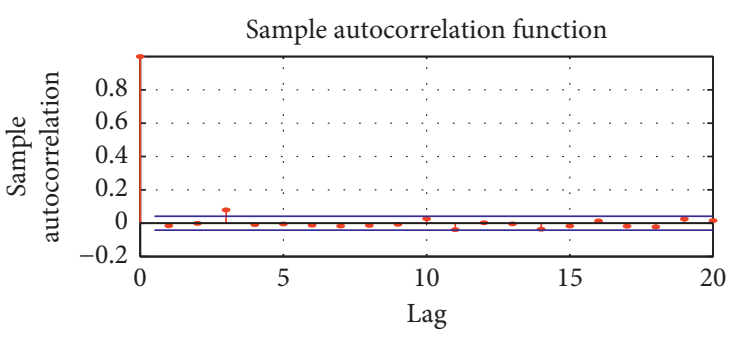

(b)

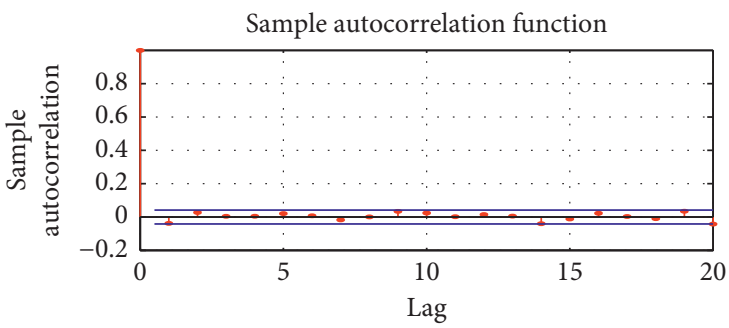

(d)

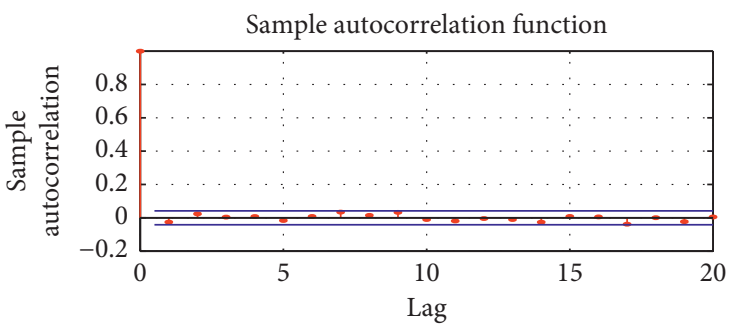

(f)

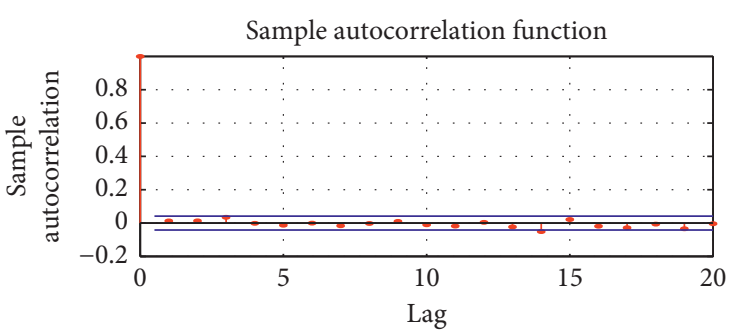

(h)

FIGURE 10: Correlograms for the four filtered log return series. (a) ACF of return for the filtered HSCEI. (b) ACF of squared return for the filtered HSCEI. (c) ACF of return for the filtered KOSPI 200. (d) ACF of squared return for the filtered KOSPI 200. (e) ACF of return for the filtered S\&P 500. (f) ACF of squared return for the filtered S\&P 500. (g) ACF of return for the filtered EURO STOXX 50. (h) ACF of squared return for the filtered EURO STOXX 50.

TABLE 3: The estimated parameters of the eight distributions and maximum log-likelihood for the filtered residuals from HSCEI's log return; No. means number of parameters; Log- $L$. means value of maximum log-likelihood.

\begin{tabular}{|c|c|c|c|c|c|}
\hline Model & No. & & & imeters & Log- $L$. \\
\hline Cauchy & 2 & $\begin{array}{c}\mu \\
0.0062\end{array}$ & $\begin{array}{c}\sigma \\
0.5493\end{array}$ & & -3531.5 \\
\hline Laplace & 2 & $\begin{array}{c}\mu \\
-0.0073\end{array}$ & $\begin{array}{c}\sigma \\
0.2773\end{array}$ & & -3260.5 \\
\hline Normal & 2 & $\begin{array}{c}\mu \\
0.0024\end{array}$ & $\begin{array}{c}\sigma \\
0.9982\end{array}$ & & -3263.1 \\
\hline Student's $t$ & 3 & $\begin{array}{c}\mu \\
0.0089\end{array}$ & $\begin{array}{c}\sigma \\
0.8539\end{array}$ & $\begin{array}{c}v \\
7.4987\end{array}$ & -3220.7 \\
\hline Skew normal & 3 & $\begin{array}{c}\mu \\
-0.0002\end{array}$ & $\begin{array}{c}\sigma \\
0.9978\end{array}$ & $\begin{array}{c}\alpha \\
-0.0006\end{array}$ & -3262.3 \\
\hline
\end{tabular}


TABLE 3: Continued.

\begin{tabular}{|c|c|c|c|c|c|c|c|}
\hline Model & No. & & & ameters & & & Log- $L$. \\
\hline Skew Cauchy & 3 & $\begin{array}{c}\mu \\
-0.0032\end{array}$ & $\begin{array}{c}\sigma \\
0.5492\end{array}$ & $\begin{array}{c}\alpha \\
0.0171\end{array}$ & & & -3531.5 \\
\hline Skew Laplace & 3 & $\begin{array}{c}\mu \\
-0.0118716\end{array}$ & $\begin{array}{c}\sigma \\
0.3788268\end{array}$ & $\begin{array}{c}\alpha \\
0.4957863\end{array}$ & & & -3260.3 \\
\hline Skew Student's $t$ & 4 & $\begin{array}{c}\mu \\
0.0715\end{array}$ & $\begin{array}{c}\sigma \\
0.9960\end{array}$ & $\begin{array}{c}\alpha \\
-0.0690\end{array}$ & $\begin{array}{c}\nu \\
7.5439\end{array}$ & & -3219.9 \\
\hline Hyperbolic & 4 & $\begin{array}{c}\mu \\
0.0694\end{array}$ & $\begin{array}{c}\sigma \\
0.9938\end{array}$ & $\begin{array}{c}\alpha \\
-0.0671\end{array}$ & $\begin{array}{c}\beta \\
2.0951\end{array}$ & & -3221.1 \\
\hline NIG & 4 & $\begin{array}{c}\mu \\
0.0718\end{array}$ & $\begin{array}{c}\sigma \\
0.944\end{array}$ & $\begin{array}{c}\alpha \\
-0.0693\end{array}$ & $\begin{array}{c}\beta \\
2.3546\end{array}$ & & -3220.6 \\
\hline Variance gamma & 4 & $\begin{array}{c}\mu \\
0.0551\end{array}$ & $\begin{array}{c}\sigma \\
0.9938\end{array}$ & $\begin{array}{c}\alpha \\
-0.0527\end{array}$ & $\begin{array}{c}\lambda \\
2.7442\end{array}$ & & -3221.7 \\
\hline G. hyperbolic & 5 & $\begin{array}{c}\mu \\
0.0564\end{array}$ & $\begin{array}{c}\sigma \\
0.996\end{array}$ & $\begin{array}{c}\alpha \\
-0.0503\end{array}$ & $\begin{array}{c}\beta \\
2.205\end{array}$ & $\begin{array}{c}\lambda \\
-1.7451\end{array}$ & -3220.4 \\
\hline
\end{tabular}

TABLE 4: The estimated parameters of the eight distributions and maximum log-likelihood for the filtered residuals from KOSPI 200's log return.

\begin{tabular}{|c|c|c|c|c|c|c|c|}
\hline Model & No. & & & Parameters & & & $\log -L$. \\
\hline Cauchy & 2 & $\begin{array}{c}\mu \\
0.0619\end{array}$ & $\begin{array}{c}\sigma \\
0.5386\end{array}$ & & & & -3517.3 \\
\hline Laplace & 2 & $\begin{array}{c}\mu \\
0.0424\end{array}$ & $\begin{array}{c}\sigma \\
-0.2793\end{array}$ & & & & -3255.9 \\
\hline Normal & 2 & $\begin{array}{c}\mu \\
-0.0040\end{array}$ & $\begin{array}{c}\sigma \\
0.9994\end{array}$ & & & & -3268.4 \\
\hline Student's $t$ & 3 & $\begin{array}{c}\mu \\
0.0276\end{array}$ & $\begin{array}{c}\sigma \\
0.8475\end{array}$ & $\begin{array}{c}v \\
6.9159\end{array}$ & & & -3230.8 \\
\hline Skew normal & 3 & $\begin{array}{c}\mu \\
-0.0149\end{array}$ & $\begin{array}{c}\sigma \\
0.9967\end{array}$ & $\begin{array}{c}\alpha \\
0.8634\end{array}$ & & & -3249.3 \\
\hline Skew Cauchy & 3 & $\begin{array}{c}\mu \\
0.1782\end{array}$ & $\begin{array}{c}\sigma \\
0.5445\end{array}$ & $\begin{array}{c}\alpha \\
-0.2173\end{array}$ & & & -3512.6 \\
\hline Skew Laplace & 3 & $\begin{array}{c}\mu \\
0.1787\end{array}$ & $\begin{array}{c}\sigma \\
0.371\end{array}$ & $\begin{array}{c}\alpha \\
0.5595\end{array}$ & & & -3249.1 \\
\hline Skew Student's $t$ & 4 & $\begin{array}{c}\mu \\
0.3423\end{array}$ & $\begin{array}{c}\sigma \\
0.9769\end{array}$ & $\begin{array}{c}\alpha \\
-0.3470\end{array}$ & $\begin{array}{c}\nu \\
8.0386\end{array}$ & & -3219.3 \\
\hline Hyperbolic & 4 & $\begin{array}{c}\mu \\
0.2849\end{array}$ & $\begin{array}{c}\sigma \\
0.9829\end{array}$ & $\begin{array}{c}\alpha \\
-0.2891\end{array}$ & $\begin{array}{c}\beta \\
1.7735\end{array}$ & & -3216.3 \\
\hline NIG & 4 & $\begin{array}{c}\mu \\
0.3056\end{array}$ & $\begin{array}{c}\sigma \\
0.9803\end{array}$ & $\begin{array}{c}\alpha \\
-0.3096\end{array}$ & $\begin{array}{c}\beta \\
2.2403\end{array}$ & & -3217.0 \\
\hline Variance gamma & 4 & $\begin{array}{c}\mu \\
0.2724\end{array}$ & $\begin{array}{c}\sigma \\
0.9841\end{array}$ & $\begin{array}{c}\alpha \\
-0.2764\end{array}$ & $\begin{array}{c}\lambda \\
2.4234\end{array}$ & & -3215.8 \\
\hline G. hyperbolic & 5 & $\begin{array}{c}\mu \\
0.2725\end{array}$ & $\begin{array}{c}\sigma \\
0.9839\end{array}$ & $\begin{array}{c}\alpha \\
-0.2764\end{array}$ & $\begin{array}{c}\beta \\
0.1516\end{array}$ & $\begin{array}{c}\lambda \\
2.4171\end{array}$ & -3215.8 \\
\hline
\end{tabular}

(ii) A plot of $\log \left(\widehat{F}\left(X_{(t)}\right)\right)$ against $X_{(t)}$ superimposed onto a plot of $\log (1 /(N+1))$ against $X_{(t)}$ shows the left tail fit for the fitted distribution

In Figure 11, the circles correspond to the empirical data, the red line corresponds to the Cauchy distribution, the red dashed line to the Laplace, the red dash-dotted line to the normal, the blue line to the Student's $t$, the blue dashed line to the skew normal, the blue dash-dotted line to the skew Cauchy, the black line to the skew Laplace, the black dashed line to the skew Student's $t$, the black dash-dotted line to the hyp, the green line to the NIG, the green dashed line to the variance gamma, and the green dash-dotted line to the ghyp distributions.

Looking to each subfigure, we chose the best-fit candidate distributions for the left tail in the return distribution for each stock index. Therefore, skew Student's $t$ and ghyp distributions were chosen for HSCEI and the hyp, and variance gamma and ghyp distributions were chosen for KOSPI 200 and S\&P 500. For EURO STOXX 50 and skew Student's $t$, hyp and ghyp were chosen.

Based on the results from information criteria, Kolmogorov-Smirnov test, and the graphical tail test, we finally chose the best-fit distribution among the 12 


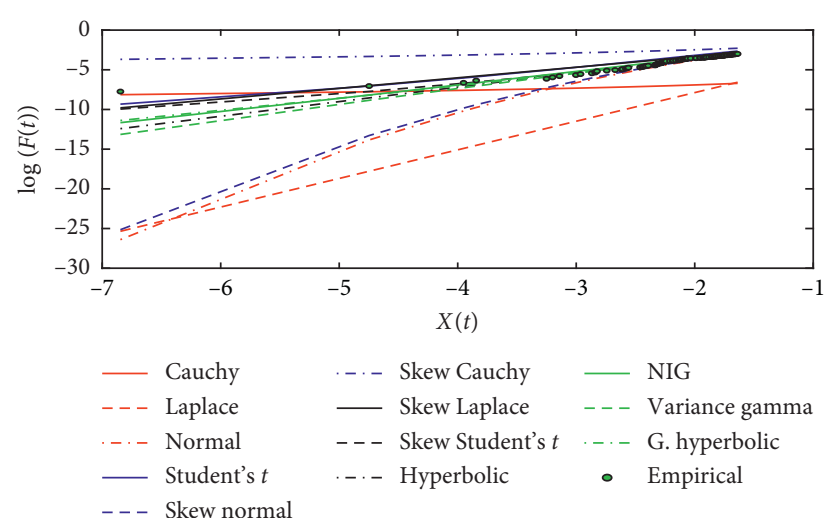

(a)

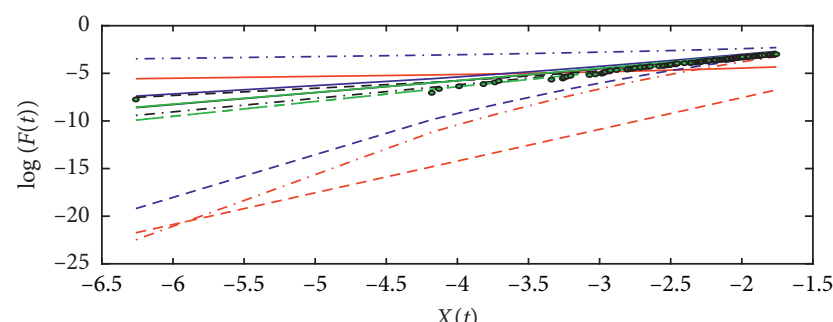

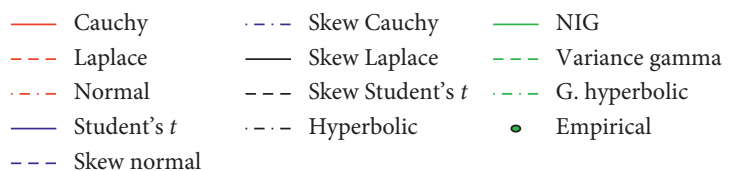

(c)

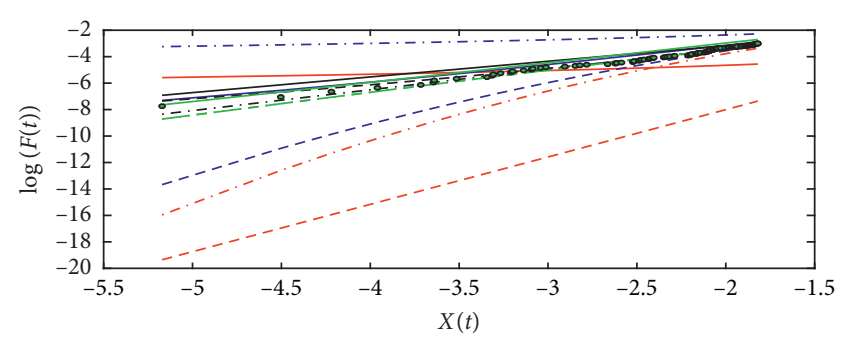

- Cauchy _..- Skew Cauchy _ NIG

- - Laplace

_ Skew Laplace _- - Variance gamma

-..- Normal

- - - Skew Student's t _... G. Gyperbolic

- Student's $t$

- - - Skew normal

(b)

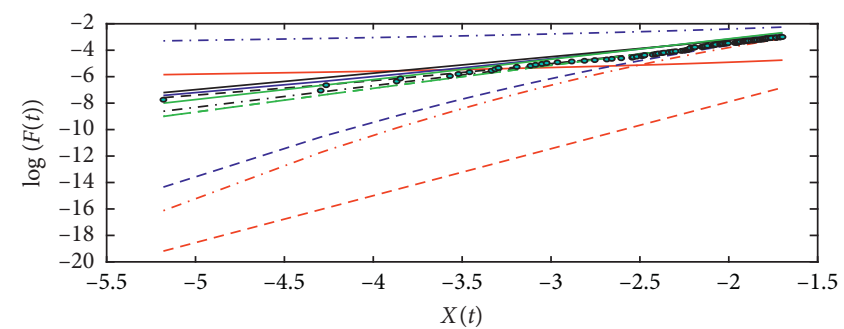

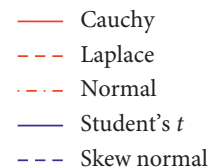

... Skew Cauchy _ NIG

_- Skew Laplace _- - Variance gamma

- - - Skew Student's t . .. - G. hyperbolic

..- Hyperbolic @ Empirical

(d)

FIgURE 11: The graphical tail test for the stock indices. (a) HSCEI. (b) KOSPI 200. (c) S\&P 500. (d) EURO STOXX 50.

TABle 5: The estimated parameters of the eight distributions and maximum log-likelihood for the filtered residuals from S\&P 500's log return.

\begin{tabular}{|c|c|c|c|c|c|c|c|}
\hline Model & No. & & & Parameters & & & $\log -L$. \\
\hline Cauchy & 2 & $\begin{array}{c}\mu \\
0.0761\end{array}$ & $\begin{array}{c}\sigma \\
0.5122\end{array}$ & & & & -3452.9 \\
\hline Laplace & 2 & $\begin{array}{c}\mu \\
0.0606\end{array}$ & $\begin{array}{c}\sigma \\
0.3003\end{array}$ & & & & -3207.6 \\
\hline Normal & 2 & $\begin{array}{c}\mu \\
0.0063\end{array}$ & $\begin{array}{c}\sigma \\
0.9985\end{array}$ & & & & -3263.2 \\
\hline Student's $t$ & 3 & $\begin{array}{c}\mu \\
0.0436\end{array}$ & $\begin{array}{c}\sigma \\
0.7991\end{array}$ & $\begin{array}{c}v \\
5.3023\end{array}$ & & & -3203.9 \\
\hline Skew normal & 3 & $\begin{array}{c}\mu \\
-0.0074\end{array}$ & $\begin{array}{c}\sigma \\
0.9955\end{array}$ & $\begin{array}{c}\alpha \\
0.8763\end{array}$ & & & -3248.5 \\
\hline Skew Cauchy & 3 & $\begin{array}{c}\mu \\
0.1819\end{array}$ & $\begin{array}{c}\sigma \\
0.5175\end{array}$ & $\begin{array}{c}\alpha \\
-0.2155\end{array}$ & & & -3447.6 \\
\hline Skew Laplace & 3 & $\begin{array}{c}\mu \\
0.1332\end{array}$ & $\begin{array}{c}\sigma \\
0.3665\end{array}$ & $\begin{array}{c}\alpha \\
0.5422\end{array}$ & & & -3200.6 \\
\hline Skew Student's $t$ & 4 & $\begin{array}{c}\mu \\
0.2421\end{array}$ & $\begin{array}{c}\sigma \\
0.9888\end{array}$ & $\begin{array}{c}\alpha \\
-0.2373\end{array}$ & $\begin{array}{c}\nu \\
5.8101\end{array}$ & & -3193.9 \\
\hline Hyperbolic & 4 & $\begin{array}{c}\mu \\
0.2062\end{array}$ & $\begin{array}{c}\sigma \\
0.9914\end{array}$ & $\begin{array}{c}\alpha \\
-0.1999\end{array}$ & $\begin{array}{c}\beta \\
0.8485\end{array}$ & & -3186.4 \\
\hline NIG & 4 & $\begin{array}{c}\mu \\
0.2259\end{array}$ & $\begin{array}{c}\lambda \\
0.9869\end{array}$ & $\begin{array}{c}\sigma \\
-0.2195\end{array}$ & $\begin{array}{c}\beta \\
1.4165\end{array}$ & & -3189.1 \\
\hline Variance gamma & 4 & $\begin{array}{c}\mu \\
0.2043\end{array}$ & $\begin{array}{c}\sigma \\
0.9886\end{array}$ & $\begin{array}{c}\alpha \\
-0.1979\end{array}$ & $\begin{array}{c}\lambda \\
1.6782\end{array}$ & & -3185.1 \\
\hline G. hyperbolic & 5 & $\begin{array}{c}\mu \\
0.2042\end{array}$ & $\begin{array}{c}\sigma \\
0.9893\end{array}$ & $\begin{array}{c}\alpha \\
-0.1982\end{array}$ & $\begin{array}{c}\beta \\
0.0781\end{array}$ & $\begin{array}{c}\lambda \\
1.6772\end{array}$ & -3185.1 \\
\hline
\end{tabular}


TABLE 6: The estimated parameters of the eight distributions and maximum log-likelihood for the filtered residuals from EURO STOXX 50's $\log$ return.

\begin{tabular}{|c|c|c|c|c|c|c|c|}
\hline Model & No. & & & Parameters & & & $\log -L$. \\
\hline Cauchy & 2 & $\begin{array}{c}\mu \\
0.0474\end{array}$ & $\begin{array}{c}\sigma \\
0.5384\end{array}$ & & & & -3513.7 \\
\hline Laplace & 2 & $\begin{array}{c}\mu \\
0.0302\end{array}$ & $\begin{array}{c}\sigma \\
0.2817\end{array}$ & & & & -3250.4 \\
\hline Normal & 2 & $\begin{array}{c}\mu \\
-0.0001\end{array}$ & $\begin{array}{c}\sigma \\
0.9959\end{array}$ & & & & -3258.3 \\
\hline Student's $t$ & 3 & $\begin{array}{c}\mu \\
0.0226\end{array}$ & $\begin{array}{c}\sigma \\
0.8485\end{array}$ & $\begin{array}{c}v \\
7.1443\end{array}$ & & & -3222.1 \\
\hline Skew normal & 3 & $\begin{array}{c}\mu \\
-0.0079\end{array}$ & $\begin{array}{c}\sigma \\
0.9944\end{array}$ & $\begin{array}{c}\alpha \\
-0.0007\end{array}$ & & & -3249.4 \\
\hline Skew Cauchy & 3 & $\begin{array}{c}\mu \\
0.1279\end{array}$ & $\begin{array}{c}\sigma \\
0.5418\end{array}$ & $\begin{array}{c}\alpha \\
-0.1494\end{array}$ & & & -3511.5 \\
\hline Skew Laplace & 3 & $\begin{array}{c}\mu \\
0.103\end{array}$ & $\begin{array}{c}\sigma \\
0.3750\end{array}$ & $\begin{array}{c}\alpha \\
0.5332\end{array}$ & & & -3247.4 \\
\hline Skew Student's $t$ & 4 & $\begin{array}{c}\mu \\
0.2383\end{array}$ & $\begin{array}{c}\sigma \\
0.9860\end{array}$ & $\begin{array}{c}\alpha \\
7.6407\end{array}$ & $\begin{array}{c}v \\
-0.2387\end{array}$ & & -3216.1 \\
\hline Hyperbolic & 4 & $\begin{array}{c}\mu \\
0.2168\end{array}$ & $\begin{array}{c}\sigma \\
0.9871\end{array}$ & $\begin{array}{c}\alpha \\
-0.2179\end{array}$ & $\begin{array}{c}\beta \\
1.7874\end{array}$ & & -3213.6 \\
\hline NIG & 4 & $\begin{array}{c}\mu \\
0.2274\end{array}$ & $\begin{array}{c}\sigma \\
0.9859\end{array}$ & $\begin{array}{c}\alpha \\
-0.2274\end{array}$ & $\begin{array}{c}\beta \\
2.2103\end{array}$ & & -3214.2 \\
\hline Variance gamma & 4 & $\begin{array}{c}\mu \\
0.2052\end{array}$ & $\begin{array}{c}\sigma \\
0.9872\end{array}$ & $\begin{array}{c}\alpha \\
-0.2052\end{array}$ & $\begin{array}{c}\lambda \\
2.4650\end{array}$ & & -3213.1 \\
\hline G. hyperbolic & 5 & $\begin{array}{c}\mu \\
0.2072\end{array}$ & $\begin{array}{c}\sigma \\
0.9861\end{array}$ & $\begin{array}{c}\alpha \\
-0.2075\end{array}$ & $\begin{array}{c}\beta \\
0.2689\end{array}$ & $\begin{array}{c}\lambda \\
2.4395\end{array}$ & -3213.1 \\
\hline
\end{tabular}

parametric distributions for each stock index, which is shown in Table 9.

\section{Risk Analysis}

In order to measure the risk, we use the value-at-risk and the conditional tail expectation given in (19) and (20), respectively.

Definition 4 (value-at-risk (VaR)). VaR at a confidence level $\theta \in(0,1)$ for loss $L$ of a security or a portfolio is defined to be

$$
\mathrm{VaR}_{\theta}=\inf \left\{l \in \mathbb{R}: F_{L}(l) \geq \theta\right\},
$$

where $F$ is the distribution function of loss.

Definition 5 (conditional tail expectation (CTE)). CTE at a confidence level $\theta \in(0,1)$ for loss $L$ of a security or a portfolio is defined to be

$$
\mathrm{CTE}_{\theta}=\mathrm{E}\left\{L \mid L<\mathrm{VaR}_{\theta}\right\} .
$$

As a means of explanation, the VaR shows how much a portfolio loses within a certain time period. By contrast, the CTE indicates the expected loss whenever the occurred loss is greater than the $\mathrm{VaR}$. In practice, the confidence level ranges from 95 to $99.5 \%$, though the Basel committee recommends $99 \%$.

4.1. Application to the Stock Index. In this section, we implement the backtest for VaR. The backtest period is 2013 to 2014. We compared the daily $\mathrm{VaR}$ estimates at the $99 \%$ confidence level. In order to forecast one-day ahead $\mathrm{VaR}$, we used 250 business days, meaning that the window size is the last 250 observations. A rolling-fixed-window scheme is used to forecast one-day ahead VaR.

According to McNeil and Frey [50], the one-day ahead $\mathrm{VaR}$ under the GARCH model is given by

$$
\operatorname{VaR}_{t+1}(\theta)=\widehat{\mu}_{t+1}+q_{\theta} \cdot \widehat{\sigma}_{t+1},
$$

where $q_{\theta}$ is the quantile at the given probability. $\widehat{\sigma}_{t+1}$ and $\widehat{\mu}_{t+1}$ are the forecast value of standard deviation and mean, respectively, estimated by the GARCH model.

As discussed by Christoffersen [51], we will use an unconditional coverage test and a conditional convergence test to determine if the model is appropriate. The unconditional coverage test attempts to determine whether the observed ratio of exceptions is consistent with the ratio of expected exceptions according to the $\mathrm{VaR}$ model. On the contrary, the conditional coverage test investigates whether the total number of exceptions is equal to the expected one and the VaR exception process is independently distributed through time. A violation of the VaR model is defined by

$$
X_{\mathrm{VaR}}(\theta):=\sum_{i=1}^{N} \mathbf{1}_{\left\{L_{i}<\operatorname{VaR}_{i} \theta\right\}},
$$

where $L_{i}$ and $\operatorname{VaR}_{i} \theta$ are the realized loss and $\operatorname{VaR}$ at $i$-th day for given all $N$ trading days.

We listed the results of the four indices from the VaR backtesting in Table 10. According to the backtest results, for the Student's $t$, skew Laplace, hyp, NIG, variance gamma, and ghyp distributions, we cannot reject the null hypothesis for both unconditional and conditional coverage tests under 
TABLE 7: AIC and BIC values for each distribution (AIC, BIC). The minimum value is shown in bold.

\begin{tabular}{lcccc}
\hline & HSCEI & KOSPI 200 & S\&P 500 & EURO STOXX 50 \\
\hline Cauchy & $(7067.0,7094.0)$ & $(7038.6,7065.6)$ & $(6909.8,6936.8)$ & $(7031.5,7058.5)$ \\
Laplace & $(6524.9,6536.4)$ & $(6515.8,6527.3)$ & $(6419.3,6430.8)$ & $(6504.7,6516.2)$ \\
Normal & $(6530.6,6542.1)$ & $(6529.1,6540.5)$ & $(6531.8,6543.2)$ & $(6520.1,6531.6)$ \\
Student's $t$ & $(\mathbf{6 4 4 7 . 0}, \mathbf{6 4 6 4 . 2 )}$ & $(6460.0,6477.2)$ & $(6412.5,6429.7)$ & $(6450.0,6467.2)$ \\
Skew normal & $(6530.6,6547.8)$ & $(6504.6,6521.9)$ & $(6502.9,6520.2)$ & $(6504.8,6522.0)$ \\
Skew Cauchy & $(7068.9,7068.9)$ & $(7031.2,7048.4)$ & $(6901.3,6901.3)$ & $(7029.0,7046.2)$ \\
Skew Laplace & $(6526.6,6543.8)$ & $(6504.3,6521.5)$ & $(6407.2,6424.4)$ & $(6500.9,6518.1)$ \\
Skew Student's $t$ & $(6447.9,6470.9)$ & $(6446.7,6469.6)$ & $(6395.8,6418.8)$ & $(6440.1,6463.1)$ \\
Hyperbolic & $(6450.1,6473.1)$ & $(6440.6,6463.6)$ & $(6380.6,6403.6)$ & $(6435.2,6458.1)$ \\
NIG & $(6449.3,6472.3)$ & $(6442.0,6465.0)$ & $(6386.2,6409.2)$ & $(6436.4,6459.4)$ \\
Variance gamma & $(6451.3,6474.3)$ & $(\mathbf{6 4 3 9 . 6}, \mathbf{6 4 6 2 . 6})$ & $(\mathbf{6 3 7 8 . 1}, \mathbf{6 4 0 1 . 0 )}$ & $(\mathbf{6 4 3 4 . 2}, \mathbf{6 4 5 7 . 2})$ \\
G. hyperbolic & $(6450.8,6471.8)$ & $(6441.6,6462.6)$ & $(6380.1,6401.1)$ & $(6436.3,6457.2)$ \\
\hline
\end{tabular}

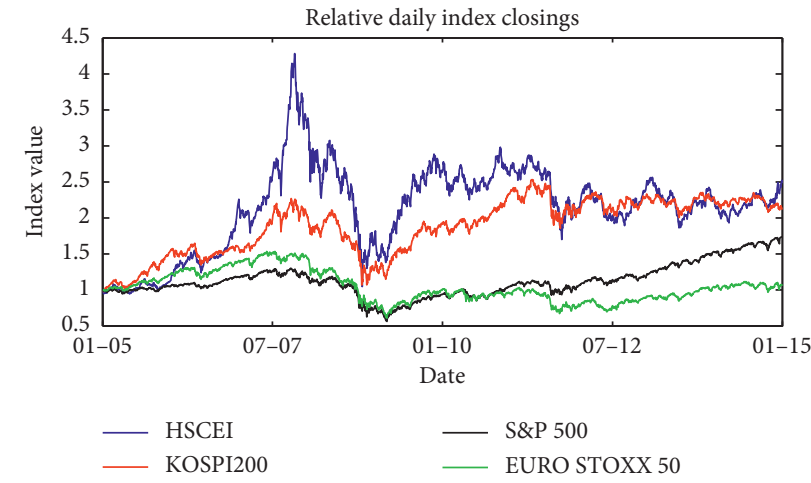

Figure 12: The relative prices of the four stock indices from 2005 to 2014.

TABLe 8: Kolmogorov-Smirnov test statistics and its $p$ values.

\begin{tabular}{|c|c|c|c|c|}
\hline & HSCEI & KOSPI 200 & S\&P 500 & EURO STOXX 50 \\
\hline $\begin{array}{l}\text { Cauchy } \\
\text { Statistic } \\
p \text { value }\end{array}$ & $\begin{array}{c}0.4404 \\
\leq 0.0000\end{array}$ & $\begin{array}{c}0.3197 \\
\leq 0.0000\end{array}$ & $\begin{array}{c}0.2891 \\
\leq 0.0000\end{array}$ & $\begin{array}{c}0.3382 \\
\leq 0.0000\end{array}$ \\
\hline $\begin{array}{l}\text { Laplace } \\
\text { Statistic } \\
p \text { value } \\
\end{array}$ & $\begin{array}{c}0.2162 \\
\leq 0.0000\end{array}$ & $\begin{array}{c}0.1999 \\
\leq 0.0000 \\
\end{array}$ & $\begin{array}{c}0.1836 \\
\leq 0.0000 \\
\end{array}$ & $\begin{array}{c}0.2035 \\
\leq 0.0000 \\
\end{array}$ \\
\hline $\begin{array}{l}\text { Normal } \\
\text { Statistic } \\
p \text { value } \\
\end{array}$ & $\begin{array}{l}0.0316 \\
0.0192 \\
\end{array}$ & $\begin{array}{c}0.0471 \\
\leq 0.0000\end{array}$ & $\begin{array}{c}0.0547 \\
\leq 0.0000\end{array}$ & $\begin{array}{c}0.0471 \\
\leq 0.0000 \\
\end{array}$ \\
\hline $\begin{array}{l}\text { Student's } t \\
\text { Statistic } \\
p \text { value } \\
\end{array}$ & $\begin{array}{l}0.0128 \\
0.8344 \\
\end{array}$ & $\begin{array}{l}0.0306 \\
0.0262 \\
\end{array}$ & $\begin{array}{l}0.0391 \\
0.0016 \\
\end{array}$ & $\begin{array}{l}0.0304 \\
0.0270 \\
\end{array}$ \\
\hline $\begin{array}{l}\text { Skew normal } \\
\text { Statistic } \\
p \text { value } \\
\end{array}$ & $\begin{array}{l}0.0308 \\
0.0241 \\
\end{array}$ & $\begin{array}{l}0.0352 \\
0.0063 \\
\end{array}$ & $\begin{array}{l}0.0460 \\
0.0001 \\
\end{array}$ & $\begin{array}{l}0.0370 \\
0.0034 \\
\end{array}$ \\
\hline $\begin{array}{l}\text { Skew Cauchy } \\
\text { Statistic } \\
p \text { value } \\
\end{array}$ & $\begin{array}{c}0.0654 \\
\leq 0.0000\end{array}$ & $\begin{array}{c}0.0667 \\
\leq 0.0000\end{array}$ & $\begin{array}{c}0.0579 \\
\leq 0.0000\end{array}$ & $\begin{array}{c}0.0642 \\
\leq 0.0000\end{array}$ \\
\hline $\begin{array}{l}\text { Skew Laplace } \\
\text { Statistic } \\
p \text { value }\end{array}$ & $\begin{array}{l}0.0381 \\
0.0023 \\
\end{array}$ & $\begin{array}{l}0.0315 \\
0.0201 \\
\end{array}$ & $\begin{array}{l}0.0201 \\
0.3013 \\
\end{array}$ & $\begin{array}{l}0.0319 \\
0.0178 \\
\end{array}$ \\
\hline $\begin{array}{l}\text { Skew Student's } t \\
\text { Statistic } \\
p \text { value }\end{array}$ & $\begin{array}{l}0.0116 \\
0.7244 \\
\end{array}$ & $\begin{array}{l}0.0143 \\
0.7244 \\
\end{array}$ & $\begin{array}{l}0.0173 \\
0.4871 \\
\end{array}$ & $\begin{array}{l}0.0184 \\
0.4080 \\
\end{array}$ \\
\hline $\begin{array}{l}\text { Hyperbolic } \\
\text { Statistic } \\
p \text { value }\end{array}$ & $\begin{array}{l}0.0132 \\
0.8060 \\
\end{array}$ & $\begin{array}{l}0.0121 \\
0.8895 \\
\end{array}$ & $\begin{array}{l}0.0102 \\
0.9674 \\
\end{array}$ & $\begin{array}{l}0.0137 \\
0.7745 \\
\end{array}$ \\
\hline
\end{tabular}


TABle 8: Continued.

\begin{tabular}{|c|c|c|c|c|}
\hline & HSCEI & KOSPI 200 & S\&P 500 & EURO STOXX 50 \\
\hline \multicolumn{5}{|l|}{ NIG } \\
\hline Statistic & 0.0371 & 0.1463 & 0.1237 & 0.1122 \\
\hline$p$ value & 0.0034 & $\leq \mathbf{0 . 0 0 0 0}$ & $\leq \mathbf{0 . 0 0 0 0}$ & $\leq \mathbf{0 . 0 0 0 0}$ \\
\hline \multicolumn{5}{|c|}{ Variance gamma } \\
\hline Statistic & 0.0136 & 0.0121 & 0.0090 & 0.0130 \\
\hline$p$ value & 0.7821 & 0.8791 & 0.9914 & 0.8225 \\
\hline \multicolumn{5}{|c|}{ G. hyperbolic } \\
\hline Statistic & 0.0114 & 0.0121 & 0.0092 & 0.013 \\
\hline$p$ value & 0.9221 & 0.8808 & 0.9879 & 0.8257 \\
\hline
\end{tabular}

TABLE 9: The best-fit distribution model for each index.

\begin{tabular}{lr}
\hline & Best-fit distribution \\
\hline HSCEI & Generalized hyperbolic \\
KOSPI 200 & Variance gamma \\
S\&P 500 & Generalized hyperbolic \\
EURO STOXX 50 & Hyperbolic \\
\hline
\end{tabular}

TABLe 10: VaR violation backtesting for four indices. $\mathscr{L}_{\mathrm{uc}}$ and $\mathscr{L}_{\mathrm{cc}}$ are the unconditional coverage test likelihood ratio statistics and the conditional coverage test likelihood ratio statistics, respectively. The Cauchy and Laplace distributions are excluded from this test because they show terrible graphical tail test for the stock indices given by Figure 11. Exp. $X_{\mathrm{VaR}}(0.01)=$ number of expected violations and Act. $X_{\mathrm{VaR}}$ $(0.01)=$ number of actual violations given $99 \% \mathrm{VaR}$ estimated.

\begin{tabular}{|c|c|c|c|c|}
\hline $\begin{array}{l}\text { Trading days }(N) \\
\text { Exp. } X_{\mathrm{VaR}}(0.01)\end{array}$ & $\begin{array}{c}\text { HSCEI } \\
489 \\
4 \\
\end{array}$ & $\begin{array}{c}\text { KOSPI } 200 \\
492 \\
4 \\
\end{array}$ & $\begin{array}{c}\text { S\&P } 500 \\
504 \\
5 \\
\end{array}$ & $\begin{array}{c}\text { EURO STOXX } 50 \\
512 \\
5 \\
\end{array}$ \\
\hline $\begin{array}{l}\text { Normal } \\
\text { Act. } X_{\mathrm{VaR}}(0.01) \\
\mathscr{L}_{\mathrm{uc}} \\
p \text { value } \\
\mathscr{L}_{\mathrm{cc}} \\
p \text { value } \\
\end{array}$ & $\begin{array}{c}9 \\
2.7955 \\
0.0945 \\
3.133 \\
0.2086 \\
\end{array}$ & $\begin{array}{c}6 \\
0.2238 \\
0.6361 \\
0.3722 \\
0.8301 \\
\end{array}$ & $\begin{array}{c}17 \\
17.7064 \\
\leq 0.0000 \\
18.895 \\
\leq 0.0000\end{array}$ & $\begin{array}{c}11 \\
5.1327 \\
0.0234 \\
5.6168 \\
0.0603 \\
\end{array}$ \\
\hline $\begin{array}{l}\text { Student's } t \\
\text { Act. } X_{\mathrm{VaR}}(0.01) \\
\mathscr{L}_{\mathrm{uc}} \\
p \text { value } \\
\mathscr{L}_{\mathrm{cc}} \\
p \text { value }\end{array}$ & $\begin{array}{c}8 \\
1.676 \\
0.1954 \\
1.9426 \\
0.3785 \\
\end{array}$ & $\begin{array}{c}5 \\
0.0013 \\
0.9711 \\
0.1041 \\
0.9492 \\
\end{array}$ & $\begin{array}{c}12 \\
6.9975 \\
0.008 \\
7.5841 \\
0.0225 \\
\end{array}$ & $\begin{array}{c}8 \\
1.3969 \\
0.2372 \\
1.6514 \\
0.4379 \\
\end{array}$ \\
\hline $\begin{array}{l}\text { Skew normal } \\
\text { Act. } X_{\mathrm{VaR}}(0.01) \\
\mathscr{L}_{\mathrm{uc}} \\
p \text { value } \\
\mathscr{L}_{\mathrm{cc}} \\
p \text { value }\end{array}$ & $\begin{array}{c}12 \\
7.43 \\
0.006 \\
8.0351 \\
0.0179\end{array}$ & $\begin{array}{c}7 \\
0.7853 \\
0.3755 \\
0.9878 \\
0.6102 \\
\end{array}$ & $\begin{array}{c}13 \\
8.8437 \\
0.002 \\
9.5336 \\
0.0085\end{array}$ & $\begin{array}{c}11 \\
5.1327 \\
0.0234 \\
5.6168 \\
0.0603\end{array}$ \\
\hline $\begin{array}{l}\text { Skew Laplace } \\
\text { Act. } X_{\mathrm{VaR}}(0.01) \\
\mathscr{L}_{\mathrm{uc}} \\
p \text { value } \\
\mathscr{L}_{\mathrm{cc}} \\
p \text { value }\end{array}$ & $\begin{array}{c}2 \\
2.221 \\
0.1361 \\
2.2375 \\
0.3266\end{array}$ & $\begin{array}{c}1 \\
4.6848 \\
0.0304 \\
4.6889 \\
0.0958\end{array}$ & $\begin{array}{c}6 \\
0.1741 \\
0.6765 \\
0.3189 \\
0.8525\end{array}$ & $\begin{array}{c}8 \\
1.3969 \\
0.2372 \\
1.6514 \\
0.4379\end{array}$ \\
\hline $\begin{array}{l}\text { Skew Student's } t \\
\text { Act. } X_{\mathrm{VaR}}(0.01) \\
\mathscr{L}_{\mathrm{uc}} \\
p \text { value } \\
\mathscr{L}_{\mathrm{cc}} \\
p \text { value }\end{array}$ & $\begin{array}{c}9 \\
2.7955 \\
0.0945 \\
3.1337 \\
0.2086\end{array}$ & $\begin{array}{c}4 \\
0.1856 \\
0.6665 \\
0.2513 \\
0.8819\end{array}$ & $\begin{array}{c}8 \\
1.4901 \\
0.222 \\
1.7487 \\
0.4171\end{array}$ & $\begin{array}{c}8 \\
1.3969 \\
0.2372 \\
1.6514 \\
0.4379\end{array}$ \\
\hline
\end{tabular}


Table 10: Continued.

\begin{tabular}{|c|c|c|c|c|}
\hline & HSCEI & KOSPI 200 & S\&P 500 & EURO STOXX 50 \\
\hline Trading days $(N)$ & 489 & 492 & 504 & 512 \\
\hline Exp. $X_{\mathrm{VaR}}(0.01)$ & 4 & 4 & 5 & 5 \\
\hline \multicolumn{5}{|l|}{ Hyperbolic } \\
\hline Act. $X_{\mathrm{VaR}}(0.01)$ & 8 & 3 & 7 & 8 \\
\hline $\mathscr{L}_{\mathrm{uc}}$ & 1.6760 & 0.8793 & 0.6867 & 1.3969 \\
\hline$p$ value & 0.1954 & 0.3483 & 0.4072 & 0.2372 \\
\hline $\mathscr{L}_{\mathrm{cc}}$ & 1.9426 & 0.9162 & 0.8843 & 1.6514 \\
\hline$p$ value & 0.3785 & 0.6324 & 0.6426 & 0.4379 \\
\hline \multicolumn{5}{|l|}{ NIG } \\
\hline Act. $X_{\mathrm{VaR}}(0.01)$ & 8 & 3 & 7 & 9 \\
\hline $\mathscr{L}_{\mathrm{uc}}$ & 1.6760 & 0.8793 & 0.6867 & 2.4230 \\
\hline$p$ value & 0.1954 & 0.3483 & 0.4072 & 0.1195 \\
\hline $\mathscr{L}_{\mathrm{cc}}$ & 1.9426 & 0.9162 & 0.8843 & 2.7457 \\
\hline$p$ value & 0.3785 & 0.6324 & 0.6426 & 0.2533 \\
\hline \multicolumn{5}{|l|}{ Variance gamma } \\
\hline Act. $X_{\mathrm{VaR}}(0.01)$ & 8 & 3 & 9 & 9 \\
\hline $\mathscr{L}_{\mathrm{uc}}$ & 1.6760 & 0.8793 & 2.5482 & 2.423 \\
\hline$p$ value & 0.1954 & 0.3483 & 0.1104 & 0.1195 \\
\hline $\mathscr{L}_{\mathrm{cc}}$ & 1.9426 & 0.9162 & 2.8761 & 2.7457 \\
\hline$p$ value & 0.3785 & 0.6324 & 0.2373 & 0.2533 \\
\hline \multicolumn{5}{|l|}{ G. hyperbolic } \\
\hline Act. $X_{\mathrm{VaR}}(0.01)$ & 8 & 3 & 9 & 9 \\
\hline $\mathscr{L}_{\mathrm{uc}}$ & 1.6760 & 0.8793 & 2.5482 & 2.423 \\
\hline$p$ value & 0.1954 & 0.3483 & 0.1104 & 0.1195 \\
\hline $\mathscr{L}_{\mathrm{cc}}$ & 1.9426 & 0.9162 & 2.8761 & 2.7457 \\
\hline$p$ value & 0.3785 & 0.6324 & 0.2373 & 0.2533 \\
\hline
\end{tabular}

TABle 11: Correlation between the filtered series.

\begin{tabular}{lcccc}
\hline Index & HSCEI & KOSPI 200 & S\&P 500 & EURO STOXX 50 \\
\hline HSCEI & 1 & 0.5950 & 0.2132 & 0.3532 \\
KOSPI 200 & 0.5950 & 1 & 0.2076 & 0.3143 \\
S\&P 500 & 0.2132 & 0.2076 & 1 & 0.6379 \\
EURO STOXX 50 & 0.3532 & 0.3143 & 0.6379 & 1 \\
\hline
\end{tabular}

TABLE 12: Risk measures for the equally weighted portfolio (direct investment) via historical data.

\begin{tabular}{lccc}
\hline Index & (HSCEI, KOSPI 200) (\%) & (S\&P 500, KOSPI 200) (\%) & (EURO STOXX 50, KOSPI 200) (\%) \\
\hline VaR (99\%) & -15.57 & -24.42 & -25.80 \\
VaR (95\%) & -12.97 & -15.87 & -18.81 \\
CTE (99\%) & -16.29 & -27.36 & -28.20 \\
CTE (95\%) & -14.59 & -20.89 & -22.70 \\
\hline
\end{tabular}

TABLE 13: Risk measures for the ELS (indirect investment) via historical data.

\begin{tabular}{lccr}
\hline Index & (HSCEI, KOSPI 200) $(\%)$ & (S\&P 500, KOSPI 200) (\%) & (EURO STOXX 50, KOSPI 200) (\%) \\
\hline VaR (99\%) & -72.68 & -55.20 & -59.52 \\
VaR (95\%) & 5.00 & 5.00 & -57.79 \\
CTE (99\%) & -74.26 & -55.84 & -59.79 \\
CTE (95\%) & 2.48 & 4.14 & -58.93 \\
\hline
\end{tabular}

confidence level 99\%. In addition, the skew Laplace, hyp, NIG, variance gamma, and ghyp have actual violations smaller than the number of expected violations for the KOSPI 200 index. Most distributions have poor backtesting results for S\&P 500. These results suggest that the optimal distribution for the risk measure of each stock index may vary.
4.2. Application to ELS. There are several kinds of ELS, for example, knock-out, bull-spread, digital-call, hi-five, and step-down. In this study, we deal with two-stock step-down ELS because this type of ELS is the best-selling ELS product on the Korean derivative market. In order to calculate the VaR and CTE for the two-stock step-down ELS, we use the 
TABLE 14: The ratio for the early repayment during the period 2005-2014.

\begin{tabular}{lccc}
\hline Redemption & (HSCEI, KOSPI 200) & (S\&P 500, KOSPI 200) & (EURO STOXX 50, KOSPI 200) \\
\hline 1st & $1156(74.39 \%)$ & $1248(80.31 \%)$ & $1136(73.10 \%)$ \\
2nd & $100(6.44 \%)$ & $64(4.12 \%)$ & $40(2.57 \%)$ \\
3rd & $101(6.5 \%)$ & $53(3.41 \%)$ & $53(3.41 \%)$ \\
4th & $72(4.63 \%)$ & $56(3.60 \%)$ & $53(3.41 \%)$ \\
th & $18(1.16 \%)$ & $65(4.18 \%)$ & $62(3.99 \%)$ \\
6th (mat) & $56(3.60 \%)$ & $46(2.96 \%)$ & $5(0.32 \%)$ \\
Loss & $51(3.28 \%)$ & $22(1.42 \%)$ & $205(13.19 \%)$ \\
\hline
\end{tabular}

TABLE 15: Risk measures for the ELS (indirect investment) via the simulation results.

\begin{tabular}{lccc}
\hline Index & (HSCEI, KOSPI 200) (\%) & (S\&P 500, KOSPI 200) (\%) & (EURO STOXX 50, KOSPI 200) (\%) \\
\hline VaR (99\%) & -69.27 & -56.20 & -73.94 \\
VaR (95\%) & -45.83 & 5.00 & -45.47 \\
CTE (99\%) & -78.08 & -65.54 & -85.09 \\
CTE (95\%) & -60.82 & -56.37 & -62.93 \\
\hline
\end{tabular}

TABLE 16: The ratio for mandatory redemption via the simulation results.

\begin{tabular}{lccc}
\hline Index & (HSCEI, KOSPI 200) & (S\&P 500, KOSPI 200) & (EURO STOXX 50, KOSPI 200) \\
\hline 1st & $35811(71.62 \%)$ & $38802(77.60 \%)$ & $35785(71.57 \%)$ \\
2nd & $4779(9.56 \%)$ & $4133(8.27 \%)$ & $4727(9.45 \%)$ \\
3rd & $2300(4.60 \%)$ & $1895(3.79 \%)$ & $2177(4.35 \%)$ \\
4th & $1921(3.84 \%)$ & $1627(3.25 \%)$ & $1953(3.91 \%)$ \\
5th & $978(1.96 \%)$ & $758(1.52 \%)$ & $900(1.80 \%)$ \\
6th (mat) & $1630(3.26 \%)$ & $1567(3.13 \%)$ & $1919(3.84 \%)$ \\
Loss & $2581(5.16 \%)$ & $1218(2.44 \%)$ & $2539(5.08 \%)$ \\
\hline
\end{tabular}

Monte Carlo simulation method. Since the two-stock stepdown ELS incorporate two-stock indices, we need to choose some pairs of stock indices. In this study, three pairs (HSCEI, KOSPI 200), (S\&P 500, KOSPI 200), and (EURO STOXX 50, KOSPI 200) are selected for the underlying assets of the twostock step-down ELS.

In this study, we consider a two-stock step-down ELS product with the following assumptions:

(i) Maturity 3 years and the valuation period is 6 months

(ii) Early redemption 90\%, 90\%, 90\%, 85\%, 85\%, and $85 \%$

(iii) Knock-in barrier 55\%

(iv) $5.0 \%$ yield (annual)

To summarize, we calculate the VaR and CTE for the two-stock step-down ELS with three kinds of pairs following the above assumptions. For the Monte Carlo simulation method, the best-fit distribution is used to generate the return distribution for each stock index. The Monte Carlo simulation uses the following steps:

(i) Generate the random variables under the best-fit distribution for each stock index

(ii) Use the Cholesky decomposition and obtain the correlated two series of random variables (iii) According to filtering equation (16), obtain the defiltered returns generated by the best-fit distribution

(iv) Calculate the VaR and CTE for the ELS

We defined a test period from 2013 to 2017 . Because the ELS with a maturity of 3 years is taken for the investigation, we considered the number of periods of 3 years in terms of 750 business days from January 1, 2013, to December 31, 2017. When counting, we considered only the closing prices of the same business day for the four stock indices.

In order to use the Cholesky decomposition, we calculated the correlations between the filtered returns from the four stock indices. The correlations are given in Table 11. All the correlations are positive, which indicates that the world economy has a similar direction of trends, although there is a difference of magnitude within them. The correlation between S\&P 500 and EURO STOXX 50 is the biggest, and the correlation between KOSPI 200 and S\&P 500 is the smallest.

Figure 12 illustrates the relative prices of the four stock indices from 2005 to 2014. In Figure 12, all the relative prices seem to change similarly. Furthermore, sharp declines during the 2008 global financial crisis are evident.

In order to enhance the robustness of the simulation results, we calculated the $\mathrm{VaR}$ and CTE for direct investments using the historical data. The direct investment is used to make an equally weighted portfolio that consists of twostock indices. That is, we consider a portfolio of two stocks 
with weights $50 \%$ and $50 \%$ having an investment period of 3 years.

The VaR and CTE for the equally weighted portfolios are given in Table 12. Furthermore, we calculate the VaR and CTE for the two-stock step-down ELS using the historical data in Table 13. Tables 12 and 13 indicate that the indirect investment, the holding of the ELS, is generally riskier than the direct investment, especially for the pair (EURO STOXX 50, KOSPI 200), where the VaR and CTE for the ELS are higher than those of direct investment given by both confidence levels.

Furthermore, we investigated the number of auto-callable cases using the historical data. The numbers and ratios of early repayment are illustrated in Table 14. From Table 14, we conclude that the first auto-callable scored over $70 \%$ for the given observations. However, the ratio for loss depends heavily on the pair of stock indices.

The VaR and CTE calculated by the Monte Carlo simulation are given in Table 15. In addition, the investigation for the auto-callable of ELS in the Monte Carlo simulation is given in Table 16. The Monte Carlo simulation results indicate that most auto-callables occur first, and the ratio of loss is approximately $5 \%$. Furthermore, from the simulation results, the inherited risk for ELS is larger than the risk calculated by the historical data.

According to the results above, the findings can be summarized as follows:

(i) Direct investments are less risky than the indirect investments in cases of ELS

(ii) Based on the historical data, the calculation of risk for ELS is inadequate to indicate the intrinsic risk of ELS to the investors

(iii) The level of risk measures for ELS depends on a pair of stock indices

\section{Summary}

The motivation for conducting this study was to discover whether these distributions are appropriate for describing the return distribution. In order to find the best-fit distributions for the four stock indices, we implemented several steps. First, we calculated the information criteria, AIC and BIC. Second, we utilized Kolmogorov-Smirnov's goodnessof-fit test. Finally, we plotted the left tails of the fitted return distribution as a graphical test. Through these steps, we could choose the best-fit distribution for each stock index.

The empirical results provide a number of interesting conclusions, with useful practical implications. Our main findings can be summarized as follows.

First, we found the best-fit distribution for each stock index. The best-fit distributions are described by the general hyperbolic distribution, which can control both the kurtosis and the skewness. Therefore, we conclude that whether the distribution describes both kurtosis and skewness is crucial to find the best-fit return distribution for a stock index.

Second, we calculated the VaR for each stock index and implemented backtesting for the estimated VaR by each distribution. These test results indicate that the distribution that has both kurtosis and skewness is adequate for estimating the VaR.

Third, based on the backtest results, the long position for ELS is riskier than the direct investment for the portfolio consisting of the same stock indices. In other words, ELS hides the inherited risk of ELS' payoff structure and attracts investors by giving the coupon higher than the return of money market.

The fourth conclusion is that the calculated measure for ELS depends on the pair of stock indices. In other words, the relationship between the two-stock indices is the main factor for risk management of ELS.

Lastly, the Monte Carlo simulation results indicate that the ELS inherit a higher risk than the risk calculated from the historical data. In general, the backtest results are presented to the investors for the sales of ELS. Therefore, it is necessary to present the simulation results regarding the risk measures to assist the rational decision of the investors.

These results have at least two implementations. First, a time-varying correlation model can be used to calculate the $\mathrm{VaR}$ for ELS because we assume that the correlation between two returns is a constant for a given period. For example, dynamic conditional correlation (Engle [52]) can be considered. Second, the hedge performance for a portfolio with different distributions can be examined. The hedging is directly related with the business profit of portfolio management. Therefore, finding the proper hedge ratio for the portfolio depending on the return distribution should be considered.

\section{Appendix}

\section{A. Parameter Estimation}

The roles of the parameters are as follows:

(i) $\mu$ : location parameter

(ii) $\sigma$ : scale parameter

(iii) $v$ : degrees of freedom in Student's $t$ distribution

(iv) $\alpha$ : skewness parameter

(v) $\beta$ : kurtosis parameter

(vi) $\lambda$ : shape parameter

\section{Data Availability}

The data used to support the findings of this study are available from the corresponding author upon request.

\section{Conflicts of Interest}

The authors declare that they have no conflicts of interest.

\section{Acknowledgments}

The work of S.-Y. Choi was supported by the Gachon University research fund of 2018 (GCU-2018-0295) and by the National Research Foundation of Korea (NRF) grant funded by the Korea government (MSIT) (no. 2019R1G1A1010278). The research of J.-H. Yoon was 
supported by the NRF of Korea grants NRF2017R1A5A1015722 and NRF-2019R1A2C108931011.

\section{References}

[1] R. Cont, "Empirical properties of asset returns: stylized facts and statistical issues," Quantitative Finance, vol. 1, no. 2, pp. 223-236, 2001.

[2] R. Huisman, R. Pownall, and K. Koedijk, "Var-x: fat tails in financial risk management," The Journal of Risk, vol. 1, no. 1, pp. 47-61, 1998.

[3] P. Verhoeven and M. McAleer, "Fat tails and asymmetry in financial volatility models," Mathematics and Computers in Simulation, vol. 64, no. 3-4, pp. 351-361, 2004.

[4] Z. Rachev, B. Rocheva-Iotovo, and S. Stoyanov, "Capturing fat tails," Risk, vol. 23, no. 5, p. 72, 2010.

[5] C.-H. Lin and S.-S. Shen, "Can the student- $t$ distribution provide accurate value at risk?" The Journal of Risk Finance, vol. 7, no. 3, pp. 292-300, 2006.

[6] E. Platen and R. Rendek, "Empirical evidence on student$t$ Log-returns of diversified world stock indices," Journal of Statistical Theory and Practice, vol. 2, no. 2, pp. 233-251, 2008.

[7] D. T. Cassidy, M. J. Hamp, and R. Ouyed, "Pricing European options with a log Student's $t$-distribution: a Gosset formula," Physica A: Statistical Mechanics and Its Applications, vol. 389, no. 24, pp. 5736-5748, 2010.

[8] A. Azzalini, "A class of distributions which includes the normal ones," Scandinavian Journal of Statistics, vol. 12, no. 2 , pp. 171-178, 1985.

[9] T. Bodnar and A. K. Gupta, "Robustness of the inference procedures for the global minimum variance portfolio weights in a skew-normal model," The European Journal of Finance, vol. 21, no. 13-14, pp. 1176-1194, 2015.

[10] M. Taniguchi, A. Petkovic, T. Kase, T. DiCiccio, and A. C. Monti, "Robust portfolio estimation under skew-normal return processes," The European Journal of Finance, vol. 21, no. 13-14, pp. 1091-1112, 2015.

[11] M. C. Jones and M. J. Faddy, "A skew extension of the $t$ distribution, with applications," Journal of the Royal Statistical Society: Series B (Statistical Methodology), vol. 65, no. 1, pp. 159-174, 2003.

[12] C. A. Abanto-Valle, V. H. Lachos, and D. K. Dey, "Bayesian estimation of a skew-student- $t$ stochastic volatility model," Methodology and Computing in Applied Probability, vol. 17, no. 3, pp. 721-738, 2015.

[13] C.-T. Gao and X.-H. Zhou, "Forecasting var and es using dynamic conditional score models and skew student distribution," Economic Modelling, vol. 53, pp. 216-223, 2016.

[14] O. E. Barndorff-Nielsen, "Normal inverse Gaussian distributions and stochastic volatility modelling," Scandinavian Journal of Statistics, vol. 24, no. 1, pp. 1-13, 1997.

[15] J. Fajardo and A. Farias, "Generalized hyperbolic distributions and brazilian data," SSRN Electronic Journal, vol. 24, no. 2, 2002.

[16] C. Necula, "Modeling heavy-tailed stock index returns using the generalized hyperbolic distribution," Romanian Journal of Economic Forecasting, vol. 10, no. 2, pp. 118-131, 2009.

[17] V. K. Socgnia and D. Wilcox, "A comparison of generalized hyperbolic distribution models for equity returns," Journal of Applied Mathematics, vol. 2014, 2014.

[18] M. Takahashi, T. Watanabe, and Y. Omori, "Volatility and quantile forecasts by realized stochastic volatility models with generalized hyperbolic distribution," International Journal of Forecasting, vol. 32, no. 2, pp. 437-457, 2016.
[19] E. Eberlein and U. Keller, "Hyperbolic distributions in finance," Bernoulli, vol. 1, no. 3, pp. 281-299, 1995.

[20] E. Eberlein, "Application of generalized hyperbolic lévy motions to finance," in Lévy Processes, pp. 319-336, Springer, Berlin, Germany, 2001.

[21] D. B. Madan and E. Seneta, "The variance gamma (V.G.) model for share market returns," The Journal of Business, vol. 63, no. 4, pp. 511-524, 1990.

[22] D. B. Madan, P. P. Carr, and E. C. Chang, "The variance gamma process and option pricing," Review of Finance, vol. 2, no. 1, pp. 79-105, 1998.

[23] A. Tjetjep and E. Seneta, "Skewed normal variance-mean models for asset pricing and the method of moments," International Statistical Review, vol. 74, no. 1, pp. 109-126, 2006.

[24] T. Moosbrucker, "Pricing CDOS with correlated variance gamma distributions," Journal of Fixed Income, vol. 12, pp. 1-30, 2006.

[25] C. Adcock, M. Eling, and N. Loperfido, "Skewed distributions in finance and actuarial science: a review," The European Journal of Finance, vol. 21, no. 13-14, pp. 1253-1281, 2015.

[26] J. Nakajima, "Bayesian analysis of multivariate stochastic volatility with skew return distribution," Econometric Reviews, vol. 36, no. 5, pp. 546-562, 2017.

[27] R. Vernic, "Multivariate skew-normal distributions with applications in insurance," Insurance: Mathematics and Economics, vol. 38, no. 2, pp. 413-426, 2006.

[28] C. Bolance, M. Guillen, E. Pelican, and R. Vernic, "Skewed bivariate models and nonparametric estimation for the CTE risk measure," Insurance: Mathematics and Economics, vol. 43, no. 3, pp. 386-393, 2008.

[29] M. Eling, "Fitting insurance claims to skewed distributions: are the skew-normal and skew-student good models?" Insurance: Mathematics and Economics, vol. 51, no. 2, pp. 239-248, 2012.

[30] M. Eling, "Fitting asset returns to skewed distributions: are the skew-normal and skew-student good models?" Insurance: Mathematics and Economics, vol. 59, pp. 45-56, 2014.

[31] K. Dowd, Beyond Value at Risk: The New Science of Risk Management, Wiley, Hoboken, NJ, USA, 1998.

[32] D. Duffie and J. Pan, "An overview of value at risk," The Journal of Derivatives, vol. 4, no. 3, pp. 7-49, 1997.

[33] W. Hu and A. Kercheval, "Risk management with generalized hyperbolic distributions," in Proceedings of the Fourth IASTED International Conference on Financial Engineering and Applications, ACTA Press, Berkeley, CA, USA, pp. 19-24, September 2007.

[34] J. A. Lopez, "Methods for evaluating value-at-risk estimates," Economic Policy Review, vol. 4, no. 3, 1998.

[35] C. Acerbi and D. Tasche, "On the coherence of expected shortfall," Journal of Banking \& Finance, vol. 26, no. 7, pp. 1487-1503, 2002.

[36] M. H. DeGroot, Optimal Statistical Decisions, Vol. 82, John Wiley \& Sons, Hoboken, NJ, USA, 2005.

[37] M. Roth, On the Multivariate $t$ Distribution, Linköping University Electronic Press, Linköping, Sweden, 2012.

[38] A. Gelman, J. B. Carlin, H. S. Stern, D. B. Dunson, A. Vehtari, and D. B. Rubin, Bayesian Data Analysis, Chapman and Hall/ CRC, London, UK, 2013.

[39] A. K. Gupta and W.-J. Huang, "Quadratic forms in skew normal variates," Journal of Mathematical Analysis and Applications, vol. 273, no. 2, pp. 558-564, 2002.

[40] A. K. Gupta, F.-C. Chang, and W.-J. Huang, "Some skewsymmetric models," Random Operators and Stochastic Equations, vol. 10, no. 2, pp. 133-140, 2002. 
[41] C. Fernandez and M. F. J. Steel, "On bayesian modeling of fat tails and skewness," Journal of the American Statistical Association, vol. 93, no. 441, pp. 359-371, 1998.

[42] H. Nurminen, T. Ardeshiri, R. Piche, and F. Gustafsson, "Robust inference for state-space models with skewed measurement noise," IEEE Signal Processing Letters, vol. 22, no. 11, pp. 1898-1902, 2015.

[43] O. Barndorff-Nielsen, "Exponentially decreasing distributions for the logarithm of particle size," Proceedings of the Royal Society of London. A. Mathematical and Physical Sciences, vol. 353, no. 1674, pp. 401-419, 1977.

[44] K. Prause, The generalized hyperbolic model: estimation, financial derivatives and risk measures, Ph.D. thesis, University of Freiburg, Breisgau, Germany, 1999.

[45] M. Abramowitz and I. A. Stegun, "Handbook of mathematical functions with formulas, graphs, and mathematical tables," National Bureau of Standards Applied Mathematics Series 55, Dover, New York, NY, USA, 1972.

[46] A. J. McNeil, R. Frey, and P. Embrechts, Quantitative Risk Management: Concepts, Techniques and Tools: Concepts, Techniques and Tools, Princeton University Press, Princeton, NJ, USA, 2015.

[47] H. Akaike, "Information theory and an extension of the maximum likelihood principle," in Selected Papers of Hirotugu Akaike, pp. 199-213, Springer, Berlin, Germany, 1998.

[48] G. Schwarz, "Estimating the dimension of a model," The Annals of Statistics, vol. 6, no. 2, pp. 461-464, 1978.

[49] K. Aas and I. H. Haff, "The generalized hyperbolic skew student's $t$-distribution," Journal of Financial Econometrics, vol. 4, no. 2, pp. 275-309, 2006.

[50] A. J. McNeil and R. Frey, "Estimation of tail-related risk measures for heteroscedastic financial time series: an extreme value approach," Journal of Empirical Finance, vol. 7, no. 3-4, pp. 271-300, nov 2000.

[51] P. F. Christoffersen, "Evaluating interval forecasts," International Economic Review, vol. 39, no. 4, pp. 841-862, 1998.

[52] R. Engle, "Dynamic conditional correlation," Journal of Business \& Economic Statistics, vol. 20, no. 3, pp. 339-350, 2002. 